\title{
Morphotropic Phase Boundary in Ferroelectric Materials
}

\author{
Abdel-Baset M. A. Ibrahim¹, Rajan Murgan², \\ Mohd Kamil Abd Rahman ${ }^{1}$ and Junaidah Osman ${ }^{3}$ \\ ${ }^{1}$ School of Physics and Material Sciences, Faculty of Applied Sciences, \\ Universiti Teknologi MARA, Selangor \\ ${ }^{2}$ Gustavus Adolphus College, Saint Peter \\ ${ }^{3}$ School of Physics, Universiti Sains Malaysia, Penang \\ 1,3 Malaysia \\ ${ }^{2}$ USA
}

\section{Introduction}

Certain solid solutions of perovskite-type ferroelectrics show excellent properties such as giant dielectric response and high electromechanical coupling constant in the vicinity of the morphotropic phase boundary (MPB). These materials are of importance to applications such as electrostrictive actuators and sensors, because of the large dielectric and piezoelectric constants (Jaffe et al., 1971; Sawaguchi, 1953; Kuwata et al., 1982; Newnham, 1997). The term "morphotropic" was originally used to refer to refer to phase transitions due to changes in composition (Ahart et al., 2008). Nowadays, the term 'morphotropic phase boundaries' (MPB) is used to refer to the phase transition between the tetragonal and the rhombohedral ferroelectric phases as a result of varying the composition or as a result of mechanical pressure (Jaffe et al., 1954; Yamashita, 1994; Yamamoto \& Ohashi, 1994; Cao \& Cross, 1993; Amin et al., 1986; Ahart et al., 2008). In the vicinity of the MPB, the crystal structure changes abruptly and the dielectric properties in ferroelectric (FE) materials and the electromechanical properties in piezoelectric materials become maximum.

The common ferroelectric materials used for MPB applications is usually complexstructured solid solutions such as lead zirconate titanate - $\mathrm{PbZr}_{1-x} \mathrm{Ti}_{x} \mathrm{O}_{3}$ (PZT) and Lead Magnesium niobate-lead titanate $\left.(1-\mathrm{x}) \mathrm{PbMg}_{1 / 3} \mathrm{Nb}_{2 / 3} \mathrm{O}_{3}-\mathrm{xPbTiO}\right)$, shortly known as PMNPT. For example, PZT is a perovskite ferroelectrics which has a MPB between the tetragonal and rhombohedral FE phases in the temperature-composition phase diagram. However, these materials are complex-structured and require a complicated and costly process to prepare its solid solutions. Furthermore, the study of the microscopic origin of its properties is very complicated.

Recently, scientists started to pay attention to the MPB in simple-structured pure compound ferroelectric materials such as ferroelectric oxides. For example, a recent experimental study on lead titanate proved that $\mathrm{PbTiO}_{3}$ can display a large MPB under pressure (Ahart et al., 2008). These experimental results even showed richer phase diagrams than those predicted by first-principle calculations. Therefore, it is of particular importance to study the 
fundamental theory of dielectric as well as piezoelectric properties of such materials in the vicinity of the MPB. Such knowledge helps engineering specific simple-structured nonlinear (NL) materials with highly nonlinear dielectric and piezoelectric properties.

Apart from first principle calculations, an alternative way to investigate the dielectric or the piezoelectric properties of these materials is to use the free energy formalism. In this chapter, we investigate the behavior of both the dynamic and the static dielectric susceptibilities in ferroelectrics in the vicinity of the MPB based on the free energy formalism. The origin of the large values of the linear and the nonlinear dielectric susceptibility tensor components is investigated using semi-analytic arguments derived from both Landau-Devonshire (LD) free energy and the Landau-Khalatnikov (LK) dynamical equation. We show that, not only the static linear dielectric constant is enhanced in the vicinity of the MPB but also the second and the third-order static nonlinear susceptibilities as well. Furthermore, the behavior of the dynamic nonlinear dielectric susceptibility as a function of the free energy parameters is also investigated for various operating frequencies. This formalism enables us to understand the enhancement of the dielectric susceptibility tensors within the concept of ferroelectric soft-modes. The input parameters used to generate the results is taken from an available experimental data of barium titanate $\mathrm{BaTiO}_{3}$ (A common simple-structured ferroelectric oxide). The effect of operating frequency, and temperature, on the dynamic dielectric susceptibility is also investigated. The enhancement of various elements of particular nonlinear optical NLO process such as second-harmonic generation (SHG) and third-harmonic generation (THG) is investigated. The enhancement of these linear and nonlinear optical processes is compared with typical values for dielectrics and ferroelectrics.

The importance of this calculation lies in the idea that the free energy material parameters $\beta_{1}$ and $\beta_{2}$ may be regarded as a function of the material composition. Therefore, this calculation can be used as one of the general guiding principles in the search for materials with large NL dielectric susceptibility coefficients. Such knowledge of MPB helps engineering specific NL materials with highly nonlinear dielectric properties. In addition, the work presented here may stimulate further interest in the fundamental theory of nonlinear response of single ferroelectric crystals with simple structure such as $\mathrm{BaTiO}_{3}$ or $\mathrm{PbTiO}_{3}$. Such pure compounds with simple structure can be used for technological applications rather than material with complicated structure.

Ishibashi \& Iwata (1998) were the first to propose a physical explanation of the MPB on the basis of a Landau-Devonshire-type of free energy with terms up to the fourth order in the polarization by adopting a "golden rule" and obtaining the Hessian matrix. They expressed the static dielectric susceptibility $\chi(\omega=0)$ in terms of the model parameters. They found that $\chi(\omega=0)$ diverges at the MPB. In the free-energy formalism, the MPB is represented by $\beta_{1}=\beta_{2}$ where $\beta_{1}$ and $\beta_{2}$ are material parameters represent the coefficients of the second and fourth-order invariants in the free energy $F$. They explained the large dielectric and piezoelectric constants in the MPB region as a result of transverse instability of the order parameter (Ishibashi \& Iwata, 1999a,b,c; Ishibashi, 2001; Iwata et al., 2002a,b). Such transverse instability is perpendicular to the radial direction in the order-parameter space near the MPB (Iwata et al., 2005). However, the work by Ishibashi et al. was limited to the study of the MPB for the static linear dielectric constant only and never extended to include the nonlinear dielectric susceptibility. Perhaps, this is because the expressions of the nonlinear 
dielectric susceptibility tensor components in terms of the free energy parameters were not yet formulated.

In earlier work by Osman et al. $\left(1998^{a, b}\right)$, the authors started to derive expressions for the nonlinear optical (NLO) susceptibilities of ferroelectric (FE) in the far infrared (FIR) spectral region based on the free energy formulation and Landau-Khalatnikov equation. The core part of this formulation is that the NLO susceptibilities are evaluated as a product of linear response functions. However, the work by Osman et al. was obtained under the approximation of a scalar polarization which only allows them to obtain specific nonlinear susceptibility elements. Soon after that, Murgan et al. (2002), presented a more general formalism for calculating all the second and third-order nonlinear susceptibility coefficients based on the Landau-Devonshire (LD) free energy expansion and the Landau-Khalatnikov (LK) dynamical equation. In their work they provided detailed results for all the nonvanishing tensor elements of the second and third -order nonlinear optical coefficients in the paraelectric, tetragonal and rhombohedral phase under single frequency approximation and second-order phase transitions.

Our aim here is then to utilize the expressions for the NLO susceptibility tensor components derived by Murgan et al (2002) to extend the study of the MPB to the second and third-order nonlinear susceptibility. Further, both the dynamic and static case is considered and an explanation based on the FE soft modes is provided. Because the expressions for the dielectric susceptibility given by Murgan et al. (2002) do not immediately relate to the MPB, we will first transform them into an alternative form that shows the explicit dependence on the transverse optical (TO) phonon mode and the longitudinal optical (LO) modes. The enhancement of the dynamic nonlinear susceptibility tensors is then investigated within the concept of the ferroelectric soft-mode with normal frequency $\omega_{T}$. Within the free energy formulation, the soft-mode $\omega_{T}$ is found to include the parameter $\left(\beta_{1}-\beta_{2}\right)$ as well as the parameter $\left(T-T_{c}\right)$.

\section{Background on morphotropic phase boundary (MPB)}

Most studies on MPB is performed on a complex structured ferroelectric or piezoelectric materials such as PZT or PZN-PT and only recently studies on simple structure pure ferroelectric materials such as $\mathrm{BaTiO} 3$ or PbTiO3 took place. In this section we will shortly review both theoretical and experimental results on the most common MPB materials and its main findings. Early experimental work on MPB focused mainly on the behavior of piezoelectric constant. This is because most of the measurements were based on diffraction which measure distortion of a unit cell. For example, Shirane \& Suzuki (1952) and Sawgushi (1953) found that PZT solid solutions have a very large piezoelectric response near the MPB region. Results of this kind are reviewed by Jaffe et al (1971) who first introduced the phrase "morphotropic phase diagram". A typical temperature-composition phase diagram for PZT is shown in Fig.1. The graph is after Noheda et al. (2000a). As shown in Fig. 1, the MPB is the boundary between the tetragonal and the rhombohedral phases and it occurs at the molar fraction compositions close to $x=0.47$. In addition, the MPB boundary is nearly vertical in temperature scale. Above the transition temperature, PZT is cubic with the perovskite structure. At lower temperature the material becomes ferroelectric, with the symmetry of the ferroelectric phase being tetragonal $\left(\mathrm{F}_{\mathrm{T}}\right)$ for Ti-rich compositions and rhombohedral $\left(\mathrm{F}_{\mathrm{R}}\right)$ for $\mathrm{Zr}$-rich compositions. Experimentally, the maximum values of the dielectric permittivity, 
piezoelectric coefficients and the electromechanical coupling factors of PZT at room temperature occur at this MPB (Jaffe et al., 1971). However, the maximum value of the remanent polarization is shifted to smaller Ti contents.

For ferroelectrics with rhombohedral and tetragonal symmetries on the two sides of the MPB, the polar axes are $(1,1,1)$ and $(0,0,1)$ (Noheda et al., 1999). The space groups of the tetragonal and rhombohedral phases ( $\mathrm{P} 4 \mathrm{~mm}$ and $\mathrm{R} 3 \mathrm{~m}$, respectively) are not symmetryrelated, so a first order phase transition is expected at the MPB. However, this has never been observed and, only composition dependence studies are available in the literature. Because of the steepness of the phase boundary, any small compositional inhomogeneity leads to a region of phase coexistence (Kakegawa et al., 1995; Mishra \& Pandey, 1996; Zhang et al., 1997; Wilkinson et al., 1998) that conceals the tetragonal-to-rhombohedral phase transition. The width of the coexistence region has been also connected to the particle size (Cao \& Cross, 1993) and depends on the processing conditions, so a meaningful comparison of available data in this region is often not possible.

Various studies (Noheda et al., 1999; Noheda et al., 2000a; Noheda et al., 2000b; Guo et al., 2000; Cox et al., 2001) have revealed further features of the MPB. High resolution x-ray powder diffraction measurements on homogeneous sample of PZT of excellent quality have shown that in a narrow composition range there is a monoclinic phase exists between the well known tetragonal and rhombohedral phases. They pointed out that the monoclinic structure can be pictured as providing a "bridge" between the tetragonal and rhombohedral structures. The discovery of this monoclinic phase led Vanderbilt \& Cohen (2001) to carry out a topological study of the possible extrema in the Landau-type expansions continued up to the twelfth power of the polarization. They conclude that to account for a monoclinic phase it is necessary to carry out the expansion to at least eight orders. It should be noted that the free energy used to produce our results for the MPB means that our results apply only to the tetragonal and rhombohedral phases, however, since these occupy most of the $\left(\beta_{1}, \beta_{2}\right)$ plane, the restriction is then not too severe.

As mentioned above, the common understanding of continuous-phase transitions through the MPB region from tetragonal to rhombohedral, are mediated by intermediate phases of monoclinic symmetry, and that the high electromechanical response in this region is related to this phase transition. High resolution $x$-ray powder diffraction measurements on poled $\mathrm{PbZr}_{1-x} \mathrm{Ti}_{x} \mathrm{O}_{3}$ (PZT) ceramic samples close to the MPB have shown that for both rhombohedral and tetragonal compositions the piezoelectric elongation of the unit cell does not occur along the polar directions but along those directions associated with the monoclinic distortion (Guo et al., 2000). A complete thermodynamic phenomenological theory was developed by Haun et al., (1989) to model the phase transitions and singledomain properties of the PZT system. The thermal, elastic, dielectric and piezoelectric parameters of ferroelectric single crystal states were calculated. A free energy analysis was used by Cao \& Cross (1993) to model the width of the MPB region. The first principles calculations on PZT have succeeded in reproducing many of the physical properties of PZT (Saghi-Szabo et al., 1999; Bellaiche \& Vanderbilt, 1999). However, these calculations have not yet accounted for the remarkable increment of the piezoelectric response observed when the material approaches its MPB. A complicating feature of the MPB is that its width is not well defined because of compositional homogeneity and sample processing conditions (Kakegawa et al., 1995). 
Another system that has been extensively studied is the $\mathrm{Pb}\left(\mathrm{Zn}_{1 / 3} \mathrm{Nb}_{2 / 3}\right) \mathrm{O}_{3}-\mathrm{PbTiO}_{3} \quad(\mathrm{PZN}-$ PT) solid solution. It is a relaxor ferroelectric with a rhombohedral to tetragonal MPB similar to PZT. It shows excellent properties for applications such as sensors and electrostrictive actuators (Kuwata et al., 1981; Kuwata et al., 1982; Iwata et al., 2002b; Cross, 1987; Cross, 1994). The giant dielectric response in relaxors and related materials is the most important properties for applications. This is because the large dielectric response means a large dielectric constant and high electromechanical coupling constant.

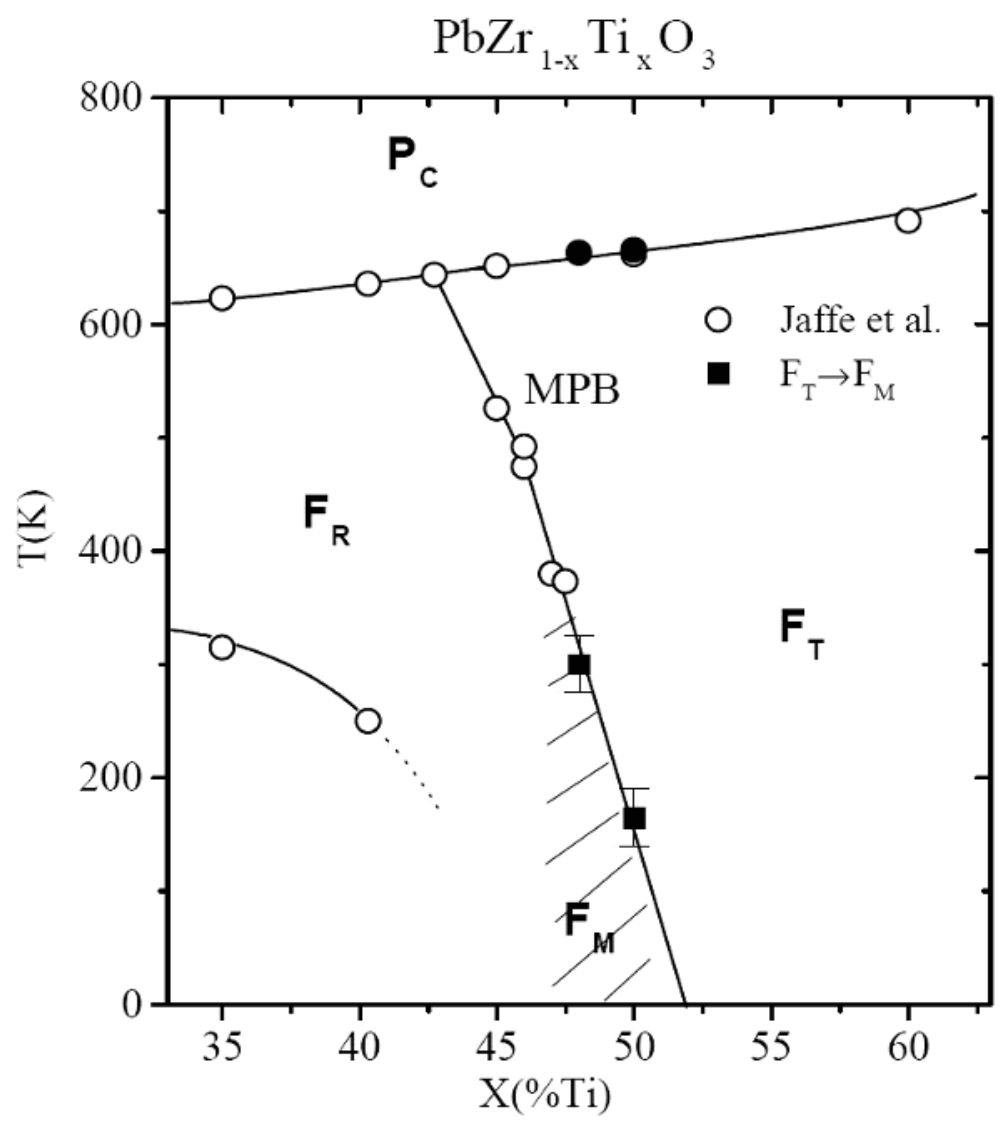

Fig. 1. The temperature-composition phase diagram for PZT where $\mathrm{P}_{\mathrm{C}}$ is the paraelectric cubic phase, $\mathrm{F}_{\mathrm{T}}$ is the ferroelectric tetragonal phase, $\mathrm{F}_{\mathrm{R}}$ is the ferroelectric rhombohedral phase and $F_{M}$ is the ferroelectric monoclinic phase. The nearly horizontal line represents the boundary between the paraelectric phase and the ferroelectric phase while the nearly vertical line represents the MPB between the tetragonal and the rhombohedral phase. The open circles represent the results obtained by Jaffe et al., (1971) while the black circles and squares represent the modifications introduced by Noheda et al., (2000a). The monoclinic phase existed at the MPB is represented by the dashed area. The graph is after Noheda et al. (2000a). 
Iwata et al. (2002b; 2005) have theoretically discussed the phase diagram, dielectric constants, elastic constants, piezoelectricity and polarization reversal in the vicinity of the MPB in perovskite-type ferroelectrics and rare-earth- $\mathrm{Fe}_{2}$ compounds based on a Landautype free energy function. They clarified that the instability of the order parameter perpendicular to the radial direction in the order-parameter space near the MPB. Such instability is induced by the isotropy or small anisotropy of the free-energy function. In addition, the transverse instability is a common phenomenon, appearing not only in the perovskite-type ferroelectric oxides, but also in magnetostrictive alloys consisting of rareearth- $\mathrm{Fe}_{2}$ compound (Ishibashi \& Iwata, 1999c), in the low-temperature phase of hexagonal $\mathrm{BaTiO}_{3}$ (Ishibashi, 2001) and in shape memory alloys (Ishibashi \& Iwata, 2003; Iwata \& Ishibashi, 2003). They also noted that the origins of the enhancement of the responses near the MPB both in the perovskite-type ferroelectrics and the rare-earth-Fe $\mathrm{F}_{2}$ compounds are the same. Even more, Iwata \& Ishibashi (2005) have also pointed out that the appearance of the monoclinic phase and the giant piezoelectric response can be explained as a consequence of the transverse instability as well.

A first principles study was done by $\mathrm{Fu} \&$ Cohen (2000) on the ferroelectric perovskite, BaTiO3, which is similar to single-crystal PZN-PT but is a simpler system to analyze. They suggested that a large piezoelectric response could be driven by polarization rotation induced by an external electric field rotation (Fu \& Cohen, 2000; Cohen, 2006). Recently, these theoretical predictions of $\mathrm{MPB}$ on a single $\mathrm{BaTiO}_{3}$ crystal have been experimentally confirmed by Ahart et al. (2008) on a pure single crystal of $\mathrm{PbTiO}_{3}$ under pressure. These results on $\mathrm{BaTiO}_{3}$ and $\mathrm{PbTiO}_{3}$ open the door for the use of pure single crystals with simple structure instead of complex materials like PZT or PMN-PT (PbMg1/3Nb2/3O3-PbTiO3) that complicates their manufacturing as well as introducing complexity in the study of the microscopic origins of their properties (Ahart et al., 2008). Moreover, Ahart et al. (2008) results on the MPB of $\mathrm{PbTiO}_{3}$ shows a richer phase diagram than those predicted by first principle calculations. It displays electromechanical coupling at the transition that is larger than any known and proves that the complex microstructures or compositions are not necessary to obtain strong piezoelectricity. This opens the door to the possible discovery of high-performance, pure compound electromechanical materials, which could greatly decrease costs and expand the utility of piezoelectric materials. For the above mentioned reasons, we are motivated here to study the NL behavior of a pure single FE with simple crystal structure such as $\mathrm{PbTiO}_{3}$ or $\mathrm{BaTiO}_{3}$ at the $\mathrm{MPB}$ on the basis of the free-energy model.

\section{The concept of morphotropic phase boundary (MPB) in the free energy}

The first published paper on modeling the MPB using the Landau-Devonshire-type of free energy was made by Ishibashi and Iwata (1998). The authors basically used the free energy $F$ as a function of the dielectric polarization in the following form;

$$
F(P)=F_{0}+\frac{\alpha}{2 \varepsilon_{0}}\left[P_{x}^{2}+P_{y}^{2}+P_{z}^{2}\right]+\frac{\beta_{1}}{4 \varepsilon_{0}^{2}}\left[P_{x}^{4}+P_{y}^{4}+P_{z}^{4}\right]+\frac{\beta_{2}}{2 \varepsilon_{0}^{2}}\left(P_{x}^{2} P_{y}^{2}+P_{y}^{2} P_{z}^{2}+P_{z}^{2} P_{x}^{2}\right)
$$

The former expression for the free energy may simply be written in the form $F=F_{0}+\Delta F$ where $F_{0}$ is the free energy is for the paraelectric phase. In Eq. (1), $\alpha$ is a temperature dependent coefficient with $\alpha=a\left(T-T_{c}\right)$ where $a$ is the inverse of the Curie 
constant, $\mathrm{T}$ is the thermodynamic temperature, and $T_{c}$ is the Curie temperature. The authors found that the static linear dielectric constant for both tetragonal and rhombohedral phases diverges at the MPB when $\beta_{1}=\beta_{2}$ in the free energy function. They proposed a phase diagram in the $T-\beta_{2} / \beta_{1}$ plane to explain the MPB as a function of the material parameters $\beta^{*}=\beta_{2} / \beta_{1}$. This diagram is reproduced in Fig. 2 for completeness. The vertical dotted line at $\beta_{1}=\beta_{2}$ represents the MPB between the rhombohedral and tetragonal phase for the static linear $\chi(\omega=0)$. The solid longitudinal line represents the boundary between the high temperature phase (Cubic) and the ferroelectric (FE) phases. It should be noted that the thermodynamic stability of the FE phases requires that $F \rightarrow \infty$ as $|\mathrm{P}| \rightarrow \infty$ for any direction of the polarization $P$. In the region of the $\beta$ plane defined by $\beta_{1}>\beta_{2}$ and $\beta_{1}+2 \beta_{2}>0$, the cubic-rhombohedral transitions of the second-order occurs. And the region defined by $\beta_{2}>\beta_{1}>0$, the cubic-tetragonal transition of the second-order occurs.

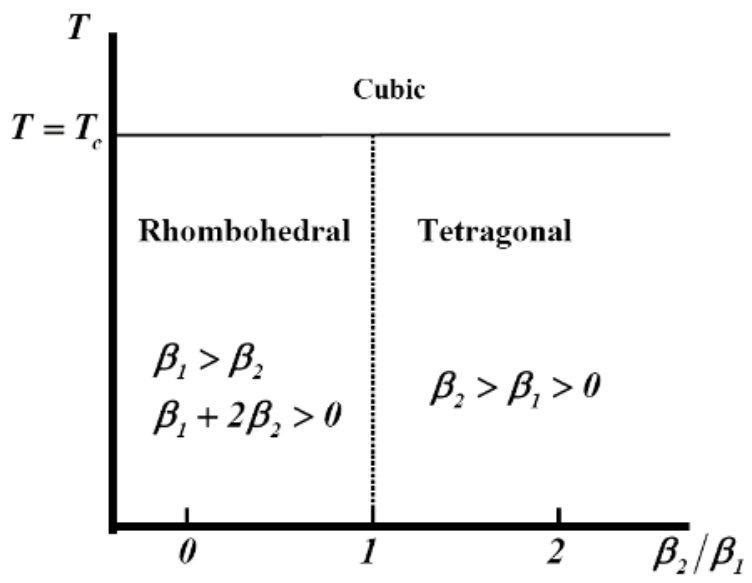

Fig. 2. The temperature-composition $\left(T-\beta_{2} / \beta_{1}\right)$ phase diagram with the vertical dotted line represents the MPB (after Ishibashi \& Iwata 1998).

In Eq. (1), if $\beta_{1}=\beta_{2}$, the free energy becomes isotropic and therefore, there is no difference between tetragonal and rhombohedral phases. To explain this, consider the polarization components $P_{x}, P_{y}$ and $P_{z}$ taken along a set of orthogonal geometrical axis and the free energy is represented by a surface where its shape depends on the value of $\beta_{1}$ and $\beta_{2}$. The case of $\beta_{1}=\beta_{2}$, the free energy is isotropic and represented by a sphere in the $x y z$ frame of reference. In the tetragonal phase the free energy surface is elongated in the direction of the spontaneous polarization to assume the shape of an ellipse (Murgan et al., 2002a). For example, if the spontaneous polarization is taken along the z-direction, therefore, the ellipsoid is elongated along this axis as seen in Fig.3 which illustrates the uniaxial nature of the tetragonal symmetry. The intersection of the isotropic surface and the tetragonal surface occurs only at the $P_{x}-P_{y}$ plane. In the rhombohedral phase, the spontaneous polarization is along the $(1,1,1)$ direction and the free energy is not only elongated in the $\mathrm{z}$-direction but also rotated as seen in Fig. 4. At the MPB the energy surface becomes isotropic but still rotated with reference to the original frame (Murgan et al., 2002a). In the previous theoretical 


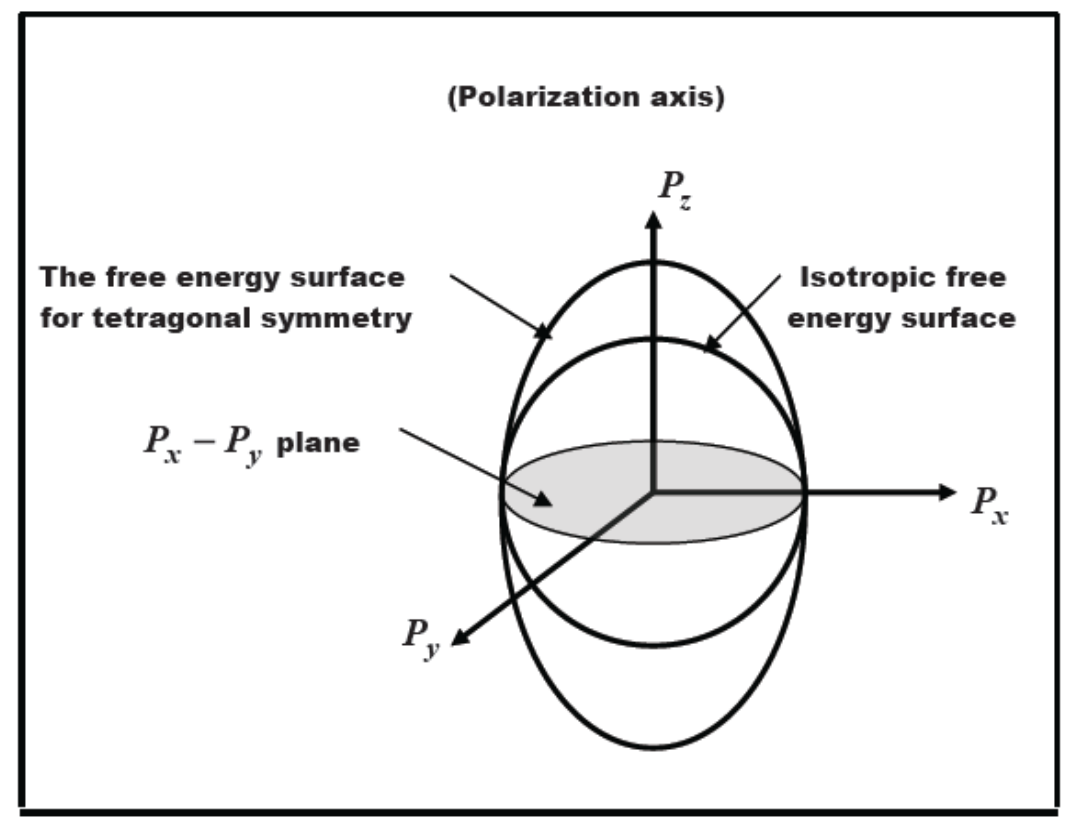

Fig. 3. The free energy surfaces for the isotropic and tetragonal systems (After Murgan et al., 2002a)

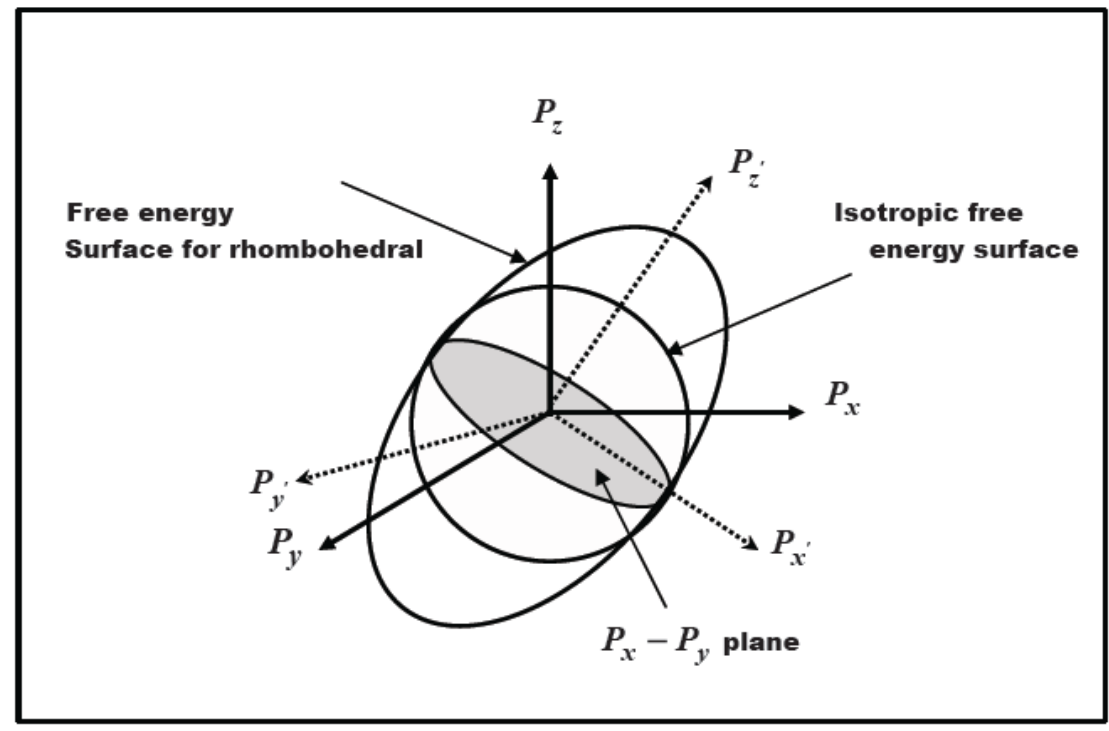

Fig. 4. The free energy surfaces for the isotropic and rhombohedral systems. ( After (Murgan et al., 2002a) 
calculations using the free energy, Haun et al., (1989) had directly related the MPB to the composition in $\mathrm{PbZrO}_{3}: \mathrm{PbTiO}_{3}$ solid solution family where the relation between the material parameters $\beta_{1}$ and $\beta_{2}$ were overlooked. In fact Ishibashi \& Iwata (1998, 1999a, 1999b) proposed that the material parameter $\beta^{*}$ may be considered a function of the mole fraction composition $x$ passing through 1 at $x=0.55$. However, the relation between the material parameters $\beta_{1}$ and $\beta_{2}$ and the composition remains a topic of further investigations.

\section{Dielectric susceptibility from Landau-Devonshire free energy}

Ishibashi \& Orihara (1994) was the first to consider the Landau-Devonshire theory to give expressions for the nonlinear dynamic dielectric response by using the Landau-Khalatnikov (LK) equation. They evaluated the NLO coefficients and the third-order nonlinear (NL) susceptibility coefficients in the paraelectric (PE) phase above the Curie temperature $T_{c}$. Subsequently, Osman et al. $\left(1998^{a, b}\right)$ have extended the theory to evaluate the NLO coefficients in the FE phase. They have demonstrated that all second order $\chi^{(2)}$ process vanishes naturally in the PE phase and that they are non zero in the FE phase due to the presence of the spontaneous polarization $\mathrm{P}_{0}$ that breaks the inversion symmetry. However, the former authors considered the free energy to be a function of a scalar polarization $P$ Soon after that, Murgan et al. (2002), used a more general form of the free energy to. calculate the dielectric susceptibility elements. In their expression, they considered the free energy expansion to be a function of a vector polarization $Q$ and additional terms were added to Eq. (1). They considered a free energy of the following form;

$$
\begin{aligned}
F(P) & =F_{0}+\frac{\alpha}{2 \varepsilon_{0}}\left[Q_{x}^{2}+Q_{y}^{2}+Q_{z}^{2}\right] \\
+ & \frac{\beta_{1}}{4 \varepsilon_{0}^{2}}\left[Q_{x}^{4}+Q_{y}^{4}+Q_{z}^{4}\right]+\frac{\beta_{2}}{2 \varepsilon_{0}^{2}}\left(Q_{x}^{2} Q_{y}^{2}+Q_{y}^{2} Q_{z}^{2}+Q_{z}^{2} Q_{x}^{2}\right)+\frac{\alpha}{2 \varepsilon_{0}}\left[P_{s}^{2}+2 Q_{z} P_{s}\right] \\
+ & \frac{\beta_{1}}{4 \varepsilon_{0}^{2}}\left[6 Q_{z}^{2} P_{s}^{2}+4 Q_{z}^{3} P_{s}+4 Q_{z} P_{s}^{3}+P_{s}^{4}\right]+\frac{\beta_{2}}{2 \varepsilon_{0}^{2}}\left[\left(Q_{z}^{2}+2 Q_{z} P_{s}+P_{s}^{2}\right)\left(Q_{x}^{2}+Q_{y}^{2}\right)\right]
\end{aligned}
$$

In the above expression, $P_{s}$ is the spontaneous polarization with its direction being along the tetragonal axes (considered in the z-direction). Eq. (2) for the free energy may simply be written in the form $F=F_{0}+\Delta F$ where $F_{0}$ is the free energy is for the paraelectric phase and the polarization components in paraelectric phase is then related to the polarization in ferroelectric tetragonal phase by $P_{x}=Q_{x}, P_{y}=Q_{y}$ and $P_{z}=Q_{z}+P_{s}$. The magnitude of the spontaneous polarization $P_{s}$ is given by the condition of minimum free energy $\partial F_{E}(P) / \partial P_{z}=0$ evaluated at $P_{z}=P_{s}$. The above expression for the free energy is more suitable for many real FE crystals that undergo successive phase transitions where additional terms are considered in comparison to Eq. (1). An important notice is that most FE, especially oxide ferroelectrics, exhibits a first-order phase transitions from the PE cubic phase to the FE phase. However, the phase transition from the cubic PE phase to the various symmetries of the lower-temperature phases can be treated as second-order provided certain conditions are fulfilled for lower symmetry groups (Haas, 1965). In the FE phase at temperatures much lower than the transition temperature, the type of transition is of no importance for the discussion of their physical properties (Ishibashi \& Iwata 1998). Together with the free energy expression in Eq. (2), LK dynamical equation $\hat{O} P_{i}=-\partial F / \partial P_{i}+E_{i}$ is 
utilized to derive various dielectric susceptibility elements (Murgan et al. (2002). The differential operator $\hat{O}=\Gamma d / d t$ is used in case of relaxational dynamics while $\hat{O}=M d^{2} / d t^{2}+\Gamma d / d t$ is used for oscillatory dynamics with $M$ and $\Gamma$ being the effective mass and the damping constant respectively.

Expressions for the second-order nonlinear susceptibility tensor elements are shown in table 1 while expressions for third-order nonlinear susceptibility tensor components are shown in Table 2. In particular, table 1 shows the nonvanishing tensor elements for second-harmonic generation (SHG) and optical rectification (OR) while Table 2 shows the nonvanishing tensor elements for the third-harmonic generation (THG) and the intensitydependent (IP) refractive index process. The expressions in both table 1 and Table 2 are all written in terms of the above linear response functions $\sigma(\omega)$ and $s(\omega)$ (Murgan et al., 2002). For SHG there are three independent elements and a total of seven nonvanishing elements while for OR there are four independent elements and a total of seven nonvanishing elements. For THG, there are five independent elements and a total of nine nonvanishing elements while for IP refractive index, there are eight independent elements and a total of a total of 15 nonvanishing elements. It should be noted that we were obliged to reproduce the results in able 1 and 2 to correct various mistakes found in the original work published by Murgan et al. (2002). The nonlinear dielectric susceptibility elements in Table 1 and 2 are given in terms of the following linear response functions in tetragonal symmetry;

\begin{tabular}{|c|c|c|}
\hline Process, and $K$ & Susceptibility $\chi^{(2)}$ & $\begin{array}{l}\text { Equation } \\
\text { Number }\end{array}$ \\
\hline \multirow{3}{*}{$\begin{array}{c}\text { SHG } \\
K=1 / 2 \\
\chi_{i l m}^{(2)}(-2 \omega ; \omega, \omega) \\
\text { Symmetric on } \\
\text { interchange } \\
\text { of }(l m)\end{array}$} & $\chi_{z z z}^{(2) S H G}=-\frac{3}{\varepsilon_{0}^{3}} \beta_{1} P_{s} s(2 \omega) s^{2}(\omega)$ & (3) \\
\hline & $\chi_{z y y}^{(2) S H G}=\chi_{z x x}^{(2) S H G}=-\frac{\beta_{2}}{\varepsilon_{0}^{3}} P_{s} s(2 \omega) \sigma^{2}(\omega)$ & (4) \\
\hline & $\chi_{x z x}^{(2) S H G}=\chi_{y z y}^{(2) S H G}=\chi_{x x z}^{(2) S H G}=\chi_{y y z}^{(2) S H G}=-\frac{\beta_{2}}{\varepsilon_{0}^{3}} P_{s} \sigma(2 \omega) \sigma(\omega) s(\omega)$ & (5) \\
\hline \multirow{4}{*}{$\begin{array}{c}\text { Optical } \\
\text { rectification (OR) } \\
K=1 / 2 \\
\chi_{\text {ilm }}^{(2)}(0 ;-\omega, \omega)\end{array}$} & $\chi_{z z z}^{(2) O R}=-\frac{3}{\varepsilon_{0}^{3}} \beta_{1} P_{s} s(0)|s(\omega)|^{2}$ & (6) \\
\hline & $\chi_{z y y}^{(2) O R}=\chi_{z x x}^{(2) O R}=-\frac{\beta_{2}}{\varepsilon_{0}^{3}} P_{s} s(0)|\sigma(\omega)|^{2}$ & (7) \\
\hline & $\chi_{x z x}^{(2) O R}=\chi_{y z y}^{(2) O R}=-\frac{\beta_{2}}{\varepsilon_{0}^{3}} P_{s} \sigma(0) s(\omega) \sigma^{*}(\omega)$ & (8) \\
\hline & $\chi_{x x z}^{(2) O R}=\chi_{y y z}^{(2) O R}=-\frac{\beta_{2}}{\varepsilon_{0}^{3}} P_{s} \sigma(0) \sigma(\omega) s^{*}(\omega)$ & (9) \\
\hline
\end{tabular}

Table 1. The nonvanishing tensor elements for second-harmonic generation (SHG) and optical rectification (OR) in ferroelectric tetragonal symmetry (Murgan et al. 2002). 


\begin{tabular}{|c|c|c|}
\hline $\begin{array}{l}\text { Process, } \\
\text { and } K\end{array}$ & Susceptibility $\chi_{i l m n}^{(3)}$ & $\begin{array}{l}\text { Eq. } \\
\text { number }\end{array}$ \\
\hline \multirow{5}{*}{$\begin{array}{c}\text { Third- } \\
\text { harmonic } \\
\text { generation } \\
\text { (THG) } \\
K=1 / 4 \\
\text { Symmetric } \\
\text { on } \\
\text { interchange } \\
\text { of }(\operatorname{lmn})\end{array}$} & $\chi_{x, x x x}^{(3) T H G}=\chi_{y, y y y}^{(3) T H G}=\frac{1}{\varepsilon_{0}^{3}} \sigma(3 \omega) \sigma^{3}(\omega)\left[\frac{2 \beta_{2}^{2} P_{s}^{2}}{\varepsilon_{0}^{2}} s(2 \omega)-\beta_{1}\right]$ & $(10)$ \\
\hline & $\chi_{z z z z}^{(3) T H G}=\frac{\beta_{1}}{\varepsilon_{0}^{3}} s(3 \omega) s^{3}(\omega)\left[\frac{18 P_{s}^{2} \beta_{1}}{\varepsilon_{0}^{2}} s(2 \omega)-1\right]$ & (11) \\
\hline & $\tilde{\chi}_{y x x y}^{(3) T H G}=\tilde{\chi}_{x x y y}^{(3) T H G}=\frac{\beta_{2}}{3 \varepsilon_{0}^{3}} \sigma(3 \omega) \sigma^{3}(\omega)\left[\frac{2 \beta_{2} P_{s}^{2}}{\varepsilon_{0}^{2}} s(2 \omega)-1\right]$ & (12) \\
\hline & $\tilde{\chi}_{x x z z}^{(3) T H G}=\tilde{\chi}_{y y z z}^{(3) T H G}=\frac{1}{3} \frac{\beta_{2}}{\varepsilon_{0}^{3}} \sigma(3 \omega) \sigma(\omega) s^{2}(\omega)\left[\frac{4 \beta_{2} P_{s}^{2} \sigma(2 \omega)+6 \beta_{1} P_{s}^{2} s(2 \omega)}{\varepsilon_{0}^{2}}-1\right]$ & (13) \\
\hline & 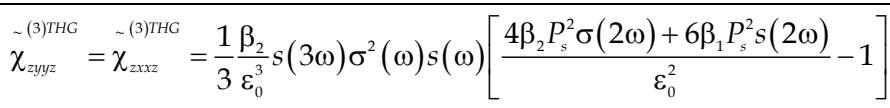 & (14) \\
\hline \multirow{8}{*}{$\begin{array}{l}\text { Intensity- } \\
\text { dependent } \\
\text { refractive } \\
\text { index (IP) } \\
K=3 / 4\end{array}$} & $\chi_{x x x x}^{(3) I P}=\chi_{y y y y}^{(3) I P}=\frac{1}{\varepsilon_{0}^{3}} \sigma^{*}(\omega) \sigma^{3}(\omega)\left[\frac{2}{\varepsilon_{0}^{2}} \beta_{2}^{2} P_{s}^{2} s(0)-\beta_{1}\right]$ & (15) \\
\hline & $\chi_{z z z z}^{(3) I P}=\frac{\beta_{1}}{\varepsilon_{0}^{3}} s^{3}(\omega) s^{*}(\omega)\left[\frac{18 \beta_{1}}{\varepsilon_{0}^{2}} P_{s}^{2} s(0)-1\right]$ & (16) \\
\hline & $\chi_{y x x y}^{(3) I P}=\chi_{x y y x}^{(3) I P}=-\frac{\beta_{2}}{3 \varepsilon_{0}^{3}} \sigma^{3}(\omega) \sigma^{*}(\omega)$ & (17) \\
\hline & $\tilde{\chi}_{x x y y}^{(3) I P}=\tilde{\chi}_{y y x x}^{(3) I P}=\frac{\beta_{2}}{2 \varepsilon_{0}^{3}} \sigma^{3}(\omega) \sigma^{*}(\omega)\left[\frac{2 \beta_{2} P_{s}^{2} s(0)}{\varepsilon_{0}^{2}}-\frac{2}{3}\right]$ & (18) \\
\hline & $\chi_{y z z y}^{(3) I P}=\chi_{x z z x}^{(3) I P}=\frac{\beta_{2}}{\varepsilon_{0}^{3}} s^{2}(\omega) \sigma^{*}(\omega) \sigma(\omega)\left[\frac{2 \beta_{2} P_{s}^{2}}{\varepsilon_{0}^{2}} \sigma(0)-\frac{1}{3}\right]$ & (19) \\
\hline & $\tilde{\chi}_{y y z z}^{(3) I P}=\tilde{\chi}_{x x z z}^{(3) I P}=\frac{\beta_{2}}{\varepsilon_{0}^{3}} \sigma^{2}(\omega) s(\omega) s^{*}(\omega)\left[\frac{3 P_{s}^{2} \beta_{1} \beta_{2} s(0)+\beta_{2} P_{s}^{2} \sigma(0)}{\varepsilon_{0}^{2}}\right]$ & (20) \\
\hline & $\chi_{z y y z}^{(3) I P}=\chi_{z x x z}^{(3) I P}=\frac{\beta_{2}}{\varepsilon_{0}^{3}} \sigma^{2}(\omega) s(\omega) s^{*}(\omega)\left[\frac{2 P_{s}^{2} \beta_{2} \sigma(0)}{\varepsilon_{0}^{2}}-\frac{1}{3}\right]$ & (21) \\
\hline & $\tilde{\chi}_{z z y y}^{(3) I P}=\tilde{\chi}_{z z x x}^{(3) I P}=\frac{\beta_{2}}{\varepsilon_{0}^{3}} s^{2}(\omega) \sigma(\omega) \sigma^{*}(\omega)\left[\frac{3 \beta_{1} P_{s}^{2} s(0)+\beta_{2} P_{s}^{2} \sigma(0)}{\varepsilon_{0}^{2}}-\frac{1}{3}\right]$ & (22) \\
\hline
\end{tabular}

Table 2. The nonvanishing tensor elements for third-harmonic generation (THG) and intensity-dependent (IP) refractive index in ferroelectric tetragonal symmetry. (Murgan et al., 2002). 


$$
\begin{gathered}
\sigma(\omega)=\left[\Theta(\omega)+\left(\alpha / \varepsilon_{0}\right)+\left(\beta_{2} P_{s}^{2} / \varepsilon_{0}^{2}\right)\right]^{-1} \\
s(\omega)=\left[\Theta(\omega)+\left(\alpha / \varepsilon_{0}\right)+\left(3 \beta_{1} P_{s}^{2} / \varepsilon_{0}^{2}\right)\right]^{-1}
\end{gathered}
$$

In the above, the frequency-dependent term is $\Theta(n \omega)=-M(n \omega)^{2}-i \Gamma(n \omega)$ for oscillatory dynamics while $\Theta(n \omega)=-i \Gamma(n \omega)$ for relaxational dynamics and $n$ is an integer number. $P_{s}^{2}$ The linear dielectric susceptibility elements $\chi_{i i}^{(1)}$ in tetragonal phase is written in terms of these uniaxial linear response functions as

$$
\chi_{x x}^{(1)}=\chi_{y y}^{(1)}=\sigma(\omega) / \varepsilon_{0} \quad, \quad \chi_{z z}^{(1)}=s(\omega) / \varepsilon_{0}
$$

where the linear dielectric susceptibility tensor $\vec{\chi}(\omega)$ is a $3 \times 3$ diagonal matrix and the average linear susceptibility may be given by $\chi_{a v}(\omega)=(1 / 3)\left(\chi_{x x}+\chi_{y y}+\chi_{z z}\right)$.

\section{The input parameters}

To plot the dielectric susceptibility, various input parameter is required. Input parameters such as $a, T_{c}$ and $M$ are taken from the available experimental data of $\mathrm{BaTiO}_{3}$ (Ibrahim et al $2007,2008,2010)$. For convenience, we may write the operating frequency $\omega$ as some coefficient $f$ multiplied by the resonance frequency $\omega_{0}$ for FE material. Thus, $f=\omega / \omega_{0}$ with $\omega_{0}$ approximated from the simple equation $\omega_{0} \approx+\sqrt{-2 a\left(T-T_{c}\right) / \varepsilon_{0} M}$ for FE materials in tetragonal phase (Ibrahim et al., 2007, 2008, 2010). The parameter $a=1 / C$ where $C=1.7 \times 10^{5} \mathrm{~K}$ is the Curie constant (Mitsui et al., 1976). This gives a value of $\omega_{0} \approx 1.4 \times 10^{14} \mathrm{~Hz}$ at room temperature. The thermodynamic temperature $T$ is fixed at room temperature.

To estimate the value of $\beta_{1}$ and $\beta_{2}$, we recall the following equation obtained by Ishibashi et al., (1998).

$$
\varepsilon_{x x}=\left[2 \beta_{1} /\left(\beta_{2}-\beta_{1}\right)\right] \varepsilon_{z z}
$$

For ferroelectric material, the dielectric constant is approximated by $\varepsilon_{x x} \approx \chi_{i i}$ since $\chi_{i i} \gg 1$. In tetragonal symmetry, expressions for both $\varepsilon_{x x}$ and $\varepsilon_{z z}$ may then be obtained by considering the static limit $\omega \rightarrow 0$ of equation (5). This gives $\varepsilon_{x x}=1 /\left[\alpha+\beta_{2} P_{s}^{2} / \varepsilon_{0}\right]$ and $\varepsilon_{z z}=1 /\left[\alpha+3 \beta_{1} P_{s}^{2} / \varepsilon_{0}\right]$ (Murgan et al., 2002). Substituting $\varepsilon_{x x}$ and $\varepsilon_{z z}$ into Eq. (26) yields the simple relation $\beta_{2}=3 \beta_{1}$. The value of $\beta_{1}$ is then estimated from the spontaneous polarization equation $P_{s}=-\varepsilon_{0} \alpha / \beta_{1}$ in tetragonal phase. This yields $\beta_{1}=7.58 \times 10^{-14} \mathrm{~m}^{3} \mathrm{~J}^{-1}$ for $P_{s} \approx 0.26 \mathrm{C} . \mathrm{m}^{-2}$ at room temperature. Hence, a value of $\beta_{2}=3 \beta_{1}=2.27 \times 10^{-13} \mathrm{~m}^{3} \mathrm{~J}^{-1}$ at room temperature. It should be noted that the value of $\beta_{1}$ and $\beta_{2}$ are very sensitive to the value of the spontaneous polarization. Estimation of the damping parameter $\Gamma$ for $\mathrm{BaTiO}_{3}$ may also be done by comparing the dielectric function in Eq. (4) and the equation $\varepsilon_{z z}(\varepsilon)=\varepsilon_{\infty}+\left[s(\omega) / \varepsilon_{0}\right]$ obtained by Osman et al. (1989a). This yields the relation;

$$
\Gamma=i M \omega-i\left[\alpha\left(1+2 \varepsilon_{0}\right) / \varepsilon_{0} \omega\left(1-\varepsilon_{0}\right)\right]
$$

which express $\Gamma$ as a function of $M, \alpha$ and $\omega$. For fixed values of $P_{s}$ and $\omega$, we have numerically found that $\Gamma$ changes by one order of magnitude (From $10^{-6}-10^{-7}$ ) over the 
range of temperature from $T=0^{\circ} \mathrm{C}$ to the $T=T_{c}$. On the other hand, the damping coefficient is relatively not a sensitive function of $P_{s}$ or $\omega$ in such a way that it maintains its order of magnitude over the relevant range of $P_{s}$ or $\omega$. A similar procedure was done by Razak et al. (2002) to estimate the damping parameter of $\mathrm{PbTiO}_{3}$. In fact, each oscillating mode in the crystal may assume different damping ratio in a real crystal and the stability of each mode depends on its damping ratio. The average damping parameter of all the relevant modes is usually obtained. However, for convenience, we have to fix the damping parameter at specific value within the range $10^{-6}-10^{-7}$ predicted by our Eq. (27). For example we may approximate the damping constant by $\Gamma \approx 3.4 \times 10^{-7} \mathrm{Kg} \cdot \mathrm{m}^{3} \cdot \mathrm{A}^{-2} \cdot \mathrm{s}^{-3}$ which corresponds to $P_{s} \approx 0.22 \mathrm{C} . \mathrm{m}^{-2}, \omega=1.01 \omega_{0}$, and $T \approx 387 \mathrm{~K}$.

\section{Morphotropic phase boundary (MPB) in linear dielectric susceptibility}

The divergence of the static dielectric susceptibility near the MPB for tetragonal and rhombohedral symmetry was first investigated by Ishibashi and Iwata (see for example Ishibashi \& Iwata 1998). They have derived the static dielectric constant $\chi(0)$ by adopting a "golden rule" and obtaining the Hessian matrix which is a $3 \times 3$ matrix composed of the second derivatives of the free energy as a function of the polarization. They found that the static limit of $\chi(\omega \rightarrow 0)$ diverges at $\beta_{1}=\beta_{2}$ in a $\chi(0)$ versus beta $\beta^{*}$ diagram where $\beta^{*}=\beta_{2} / \beta_{1}$. Especially for tetragonal symmetry, the $\chi(0)$ diverges from the right side toward $\beta^{*}=1$ (See Fig. 2).

In this section, we investigate the dynamic linear dielectric susceptibility $\chi(\omega)$ and its MPB for FE material in tetragonal phase. We will study the effect of operating frequency $\omega$ on the dynamic linear susceptibility as a function of the material parameters $\beta_{1}$ and $\beta_{2}$. Therefore, the divergence of the static limit of the linear dielectric susceptibility $\chi(0)$ at the MPB $\left(\beta_{1}=\beta_{2}\right)$ is regarded as a special case. In the static limit, the results obtained here for the static $\chi(0)$ shows similar divergence as those obtained by Ishibashi et al. (1998). To explain the behavior of $\chi(\omega)$ in terms of FE soft modes, it is necessary to write $\chi(\omega)$ in the following form;

$$
\begin{gathered}
\chi_{x x}^{(1)}=\chi_{y y}^{(1)}=\left\{M \varepsilon_{0}\left[-\omega^{2}-i \omega(\Gamma / M)+\omega_{T O}^{2}\right]\right\}^{-1} \\
\chi_{z z}^{(1)}=\left[\varepsilon_{0} M\left(-\omega^{2}-i \omega(\Gamma / M)+\omega_{L O}^{2}\right)\right]^{-1}
\end{gathered}
$$

We note that in deriving equations (27) and (28), we have used the spontaneous polarization for tetragonal phase defined by $P_{s}^{2}=-\varepsilon_{0} \alpha / \beta_{1}$. In the above equations, $\chi^{(1)}$ is written in terms of the lattice-vibrational modes, particularly, $\chi_{x x}^{(1)}$ is written in terms of the transverse-optical (TO) mode characterized by its normal frequency $\omega_{T O}^{2}=a\left(T-T_{c}\right)\left(\beta_{1}-\beta_{2}\right) / M \varepsilon_{0} \beta_{1}$ and $\chi_{z z}^{(1)}$ is written in terms of its longitudinal-optical (LO) mode $\omega_{L O}^{2}=2 a\left(T-T_{c}\right) / \varepsilon_{0} M$. The TO mode corresponds to the displacement of the free energy perpendicular to the polar axis while the LO mode corresponds to the displacement along the polar axis. Upon using $\alpha=a\left(T-T_{c}\right)$ the pole position can be determined by the soft-mode frequency.

Since the stability region of the tetragonal phase lies at $\beta_{2}>\beta_{1}$, the value of $\omega_{\text {TO }}^{2}$ is positive. As anticipated, the soft-mode frequency shows that the term $\beta_{1}-\beta_{2}$ enters on the same 
footing as the term $\left(T-T_{c}\right)$ which make the dielectric susceptibility diverges either when $T$ approaches $T_{c}$ or when $\beta_{1}$ approaches $\beta_{2}$. Therefore, $\omega_{\text {то }}$ has a double soft-mode character and the explicit limits are then $\omega_{\text {ТО }} \rightarrow 0$ as $\beta_{2} \rightarrow \beta_{1}$ or $T \rightarrow T_{c}$ and $\omega_{\text {ТО }} \rightarrow \infty$ as $\beta_{1} \rightarrow 0$ (instability limit). The reason for the instability can be seen from the spontaneous polarization $P_{s}^{2}=-\varepsilon_{0} \alpha / \beta_{1}$ where near the instability limit $P_{s}$ becomes large and therefore $\omega_{\text {TO }}$. It is important to notice that the instability limit is a kind of an artifact; it results mainly due to the truncation of the free energy to the fourth order in the polarization. Thus, when $\beta_{1}$ is very small, at least the sixth order terms in the polarization should be added to the free energy to avoid the instability.

Now the origin of the enhancement of the dielectric susceptibility is clear, when $\beta_{2} \rightarrow \beta_{1}$, the value of the soft-mode frequency $\omega_{\text {TO }}$ becomes smaller which leads to a direct enhancement of the values of $\chi_{x x}^{(1)}=\chi_{y y}^{(1)}$ as seen in equation (27). The static dielectric constant can then be derived by setting $\omega=0$ in equations (27) and (28). This leads to a similar form to those equations obtained by Ishibashi et al. (1998). These are;

$$
\begin{gathered}
\chi_{x x}^{(1)}=\chi_{y y}^{(1)}=\beta_{1} /\left[a\left(T-T_{c}\right)\left(\beta_{1}-\beta_{2}\right)\right]=1 / m \varepsilon_{0} \omega_{T O}^{2} \\
\chi_{z z}^{(1)}=-1 /\left[2 a\left(T-T_{c}\right)\right]=-1 / \varepsilon_{0} m \omega_{L O}^{2}
\end{gathered}
$$

In Eq. (29) and (30), the static linear dielectric constant shows that at the MPB, $\chi_{x x}^{(1)}(\omega \rightarrow 0)$ and $\chi_{y y}^{(1)}(\omega \rightarrow 0)$ diverge when $\beta_{1}=\beta_{2}$ at all temperatures while $\chi_{z z}^{(1)}(\omega \rightarrow 0)$ diverges only at $T \rightarrow T_{c}$. In Fig. 5(a), we plot the complex dynamic dielectric susceptibility $\chi_{x x}^{(1)}$ versus $\beta^{*}=\beta_{2} / \beta_{1}$ at single operating frequency $f=\omega / \omega_{0}=0.1$. A part from the element $\chi_{z z}^{(1)}$ which remains constant over $\beta^{*}$ because it is a function of the LO mode only, the other element $\chi_{x x}^{(1)}$ shows a resonance-like behavior at certain value of $\beta^{*}$. At this peak, the dynamic response of the dielectric susceptibility is maximized. In a way, this resonance-like behavior is a function of the material composition through the parameter $\beta^{*}$ and it is explainable within the concept of the ferroelectric soft-mode dynamics. We have numerically found that the value of $\left|\chi_{x x}^{(1)}\right|$ at its maximum is $2.4 \times 10^{4}$ which give a linear refractive index $n=\sqrt{\operatorname{Re}\left(\varepsilon_{x x}(\omega)\right)} \approx 109$ at room temperature. Meanwhile, far from the pole, at $\beta^{*}=3$, the dielectric constant is about 800 which results in a linear refractive index of 2.46 . In fact, the value of the dielectric susceptibility decreases gradually from its maximum by increasing the value of $\beta^{*}$. The values of the dielectric constant obtained here for ferroelectric materials are huge in comparison to typical dielectrics or semiconductors. For amorphous dielectrics such as fused silica, the dielectric constant is in the range 2.5-3.5 while the linear refractive index is about 1.46. In typical semiconductors such as GaAs, the dielectric constant is about 13.2 and the linear refractive index is 3.6 (Glass, 1987).

To examine the effect of operating frequency, we plot the average value of the dynamic linear dielectric susceptibility versus $\beta^{*}$ for different operating frequencies $f$ (Fig. $5(b)$ ). Other parameters kept unchanged. The general feature of these curves is that they all show a peak behavior where the dynamic linear susceptibility is maximum at certain value of $\beta^{*}$. This pole response is a strong function of the operating frequency. For example, curve $(i)$ shows the linear susceptibility $\chi_{a v}^{(1)}(\omega)$ versus $\beta^{*}$ for $f=2$, this gives a maximum value of $\chi_{a v}^{(1)}(\omega) \simeq 796$ at $\beta^{*}=9$. Curve (ii) shows the linear susceptibility $\chi_{a v}^{(1)}(\omega)$ for $f=1.5$, this gives 
a maximum value of $\chi_{a v}^{(1)}(\omega) \simeq 1060$ at $\beta^{*}=5.5$. Upon decreasing the operating frequency further to $f=1.1$ (Curve (iii)), the linear susceptibility $\chi_{a v}^{(1)}(\omega)$ assumes a maximum value of 1447 at $\beta^{*}=3.42$. Below the resonance, at $f=0.95$ (Curve $(i v)$ ), $\chi_{a v}^{(1)}(\omega)$ assumes a maximum value of 1676 at $\beta^{*}=2.81$. Far below the resonance, at $f=0.5$ (Curve $(v)$ ), $\chi_{a v}^{(1)}(\omega)$ gives a maximum value of 3185 at $\beta^{*}=1.5$. Finally, at $f=0.0$ (Curve $(v i)$ ), the static limit of the linear susceptibility $\chi_{a v}^{(1)}(0)$ in this case diverges at $\beta^{*}=1$. This result for the static case coincide with the results obtained by Ishibashi (1998) using the Hessian matrix of the free energy. Therefore, we may generally conclude that, for the dynamic dielectric susceptibility, a systematic decrease of the operating frequency $\omega=f \omega_{0}$ is accompanied by systematic enhancement of the linear dielectric susceptibility especially at its peak. However, decreasing the operating frequency is also accompanied a systematic decrease of $\beta^{*}$ towards $\beta^{*}=1$. At $\omega=0$, the value of $\chi_{a v}^{(1)}(0)$ goes to infinity at $\beta^{*}=1$ since the soft-mode frequency $\omega_{T O}$ becomes zero.

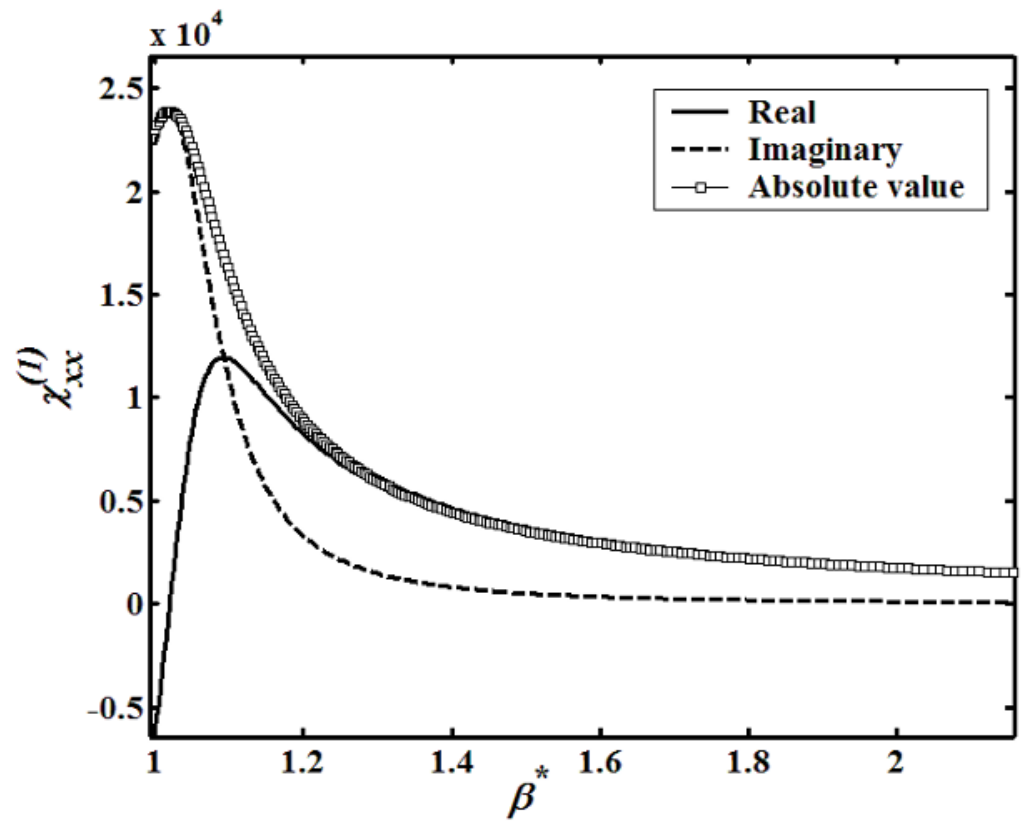

Fig. 5. (a) Linear dynamic dielectric susceptibility $\chi_{x x}^{(1)}$ versus $\beta^{\star}=\beta_{2} / \beta_{1}$ in tetragonal phase at room temperature and operating frequencies $f=\omega / \omega_{0}=0.1$.

\section{Morphotropic phase boundary in second-order nonlinear susceptibility}

In the free energy formalism, there is only one underlying dynamic equation and the NLO coefficients take the form of products of linear response functions. This formalism does not explicitly show the dependence of the NL susceptibility on the MPB or the ferroelectric soft mode. As shown in Table 1 and table 2, the susceptibility elements takes the form of a product of linear response functions, $s(n \omega)$ if the related suffix is $z$, and $\sigma(n \omega)$ if it is $x$ or $y$ 
and the argument is the related frequency. In this case, it is convenient to transform the second-order NL susceptibility tensor elements to an alternative form that shows a direct dependence on the lattice-vibrational modes.

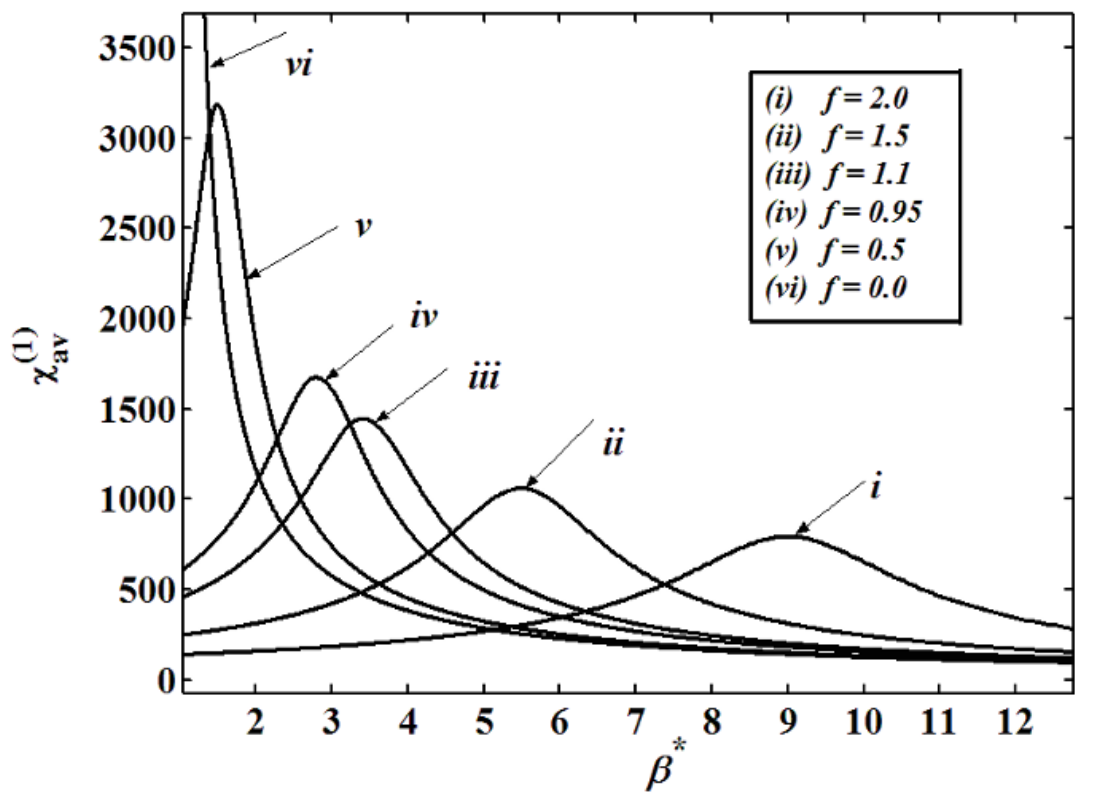

Fig. 5. (b) Linear dynamic dielectric susceptibility $\chi_{a v}^{(1)}$ versus $\beta^{*}=\beta_{2} / \beta_{1}$ in tetragonal phase at room temperature for different operating frequencies $f=\omega / \omega_{0}$ shows the improvement of the $\chi_{a v}^{(1)}(\omega)$ at the MPB.

These are the transverse-optical modes (TO) with normal frequency $\omega_{\text {То }}$ and the longitudinal-optical (LO) mode with frequency $\omega_{L O}$. In this case, the ferroelectric soft mode is one of these transverse-optical modes that soften when the thermodynamic temperature $T$ approaches $T_{c}$ or $\beta^{*}$ approaches 1 . For example, The SHG element $\chi_{z y y}^{(2) S H G}$ may be expressed explicitly in terms of these modes in the following form;

$$
\chi_{z y y}^{(2) S H G}=\chi_{z x x}^{(2) S H G}=-\beta_{2} P_{s} /\left\{\varepsilon_{0}^{3} M^{3}\left[\frac{\Theta(2 \omega)}{M}-\omega_{L O}^{2}\right]\left[\frac{\Theta(\omega)}{M}+\omega_{\text {TO }}^{2}\right]^{2}\right\}
$$

This is achieved by substituting the linear response functions $\sigma(\omega)$ and $s(\omega)$ in tetragonal symmetry from Eq. (3) and Eq. (4) into Eq. (7) and performing a series of algebraic manipulations. It should be noticed that the linear response functions for the second harmonics $\sigma(2 \omega) s(2 \omega)$ in Eq. (33) is responsible for the appearance of the function argument $\Theta(2 \omega)$ where $\Theta(2 \omega)=-M(2 \omega)^{2}-i \gamma(2 \omega)$. The static limits are then obtained by setting $\omega=0$ in Eq. (32) and performing further algebraic simplifications. The above secondorder coefficient in Eq. (32) contains a tensor suffix corresponds to the output wave of frequency $2 \omega$, and others correspond to the input of frequency $\omega$. 


$$
\chi_{z y y}^{(2) S H G}(\omega=0)=\chi_{z x x}^{(2) S H G}=\chi_{x z x}^{(2) S H G}=\beta_{2} P_{s} / \varepsilon_{0}^{3} M^{3} \omega_{T O}^{4} \omega_{L O}^{2}
$$

The element $\chi_{z y y}^{(2) S H G}$ in Eq. (32) contains one longitudinal optical mode $\omega_{L O}^{2}=2 \alpha / \varepsilon_{0} M$ corresponds to the output wave of frequency $2 \omega$ and one TO mode with frequency $\omega_{\text {TO }}^{2}=2 \alpha\left(\beta_{1}-\beta_{2}\right) / \varepsilon_{0} M \beta_{1}$ corresponds to the input frequency $\omega$. Therefore, this element is expected to assume one peak at certain value of $\beta^{*}$. This expectation is assured upon plotting $\left|\chi_{z y y}^{(2) S H G}\right|$ versus $\beta^{*}$ for different operating frequencies as shown in Fig. 6(a). The main feature of those curves is that they all display a single pole at certain value of $\beta^{*}$. In the static case (curve $i$ ), the value of $\left|\chi_{z y y}^{(2) S H G}(\omega \rightarrow 0)\right|$ increases systematically then diverges at the MPB between the tetragonal and rhombohedral phase where $\beta^{*}=1$. As seen from Eq. (33), the static value of $\left|\chi_{2 y y}^{(2) S H G}(\omega \rightarrow 0)\right|$ only depends on $\omega_{L O}$ and $\omega_{T O}$. Since $\omega_{L O}$ is not a function of $\beta^{*}$, thus the enhancement in the values of $\left|\chi_{z y y}^{(2) S H G}(\omega \rightarrow 0)\right|$ is mainly due to $\omega_{\text {TO }}$. The value of $\omega_{T O}^{2}=2 \alpha\left(\beta_{1}-\beta_{2}\right) / \varepsilon_{0} M \beta_{1}$ decreases "softens" systematically when $\beta_{1}$ approaches $\beta_{2}$ which lead to a direct increment in the value of $\left|\chi_{z y y}^{(2) S H G}(\omega \rightarrow 0)\right|$ till the divergence occur at $\beta_{1} \rightarrow \beta_{2}$ as $\omega_{\text {то }} \rightarrow 0$ at the MPB. It should be noted that, the association of a high static dielectric constant with a specific low frequency optical mode is experimentally observed on FE materials such as strontium titanate, $\mathrm{SrTiO}_{3}$ (Kittel 1995; Sirenko et al., 2000; Katayama et al., 2007).

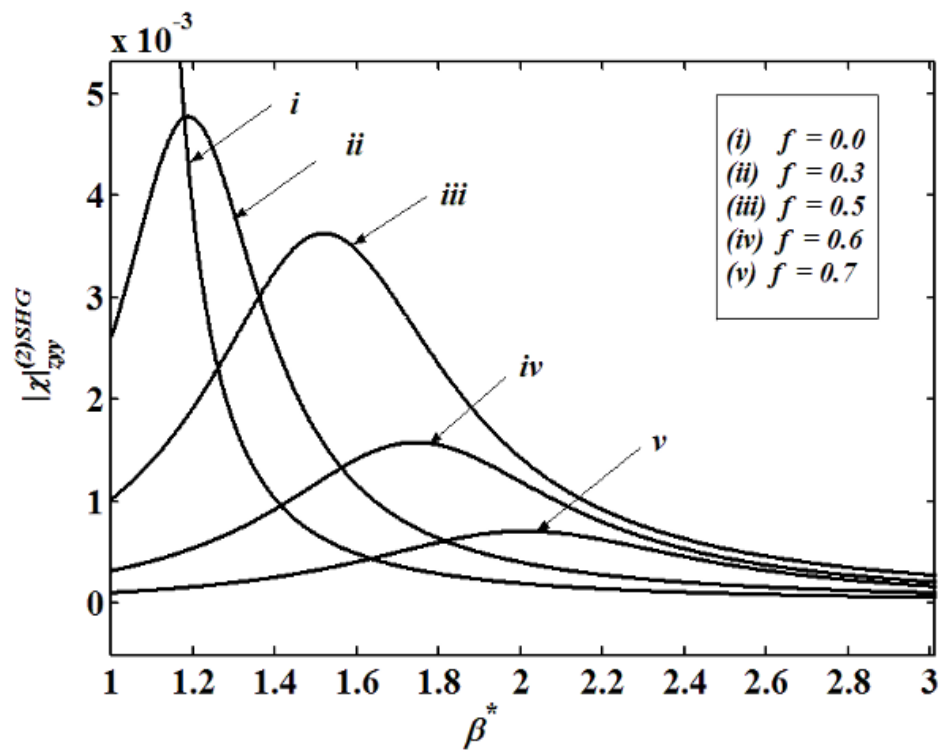

Fig. 6. (a) The nonlinear dielectric susceptibility $\chi_{z y y}^{(2) S H G}(-2 \omega ; \omega, \omega)$ versus $\beta^{*}=\beta_{2} / \beta_{1}$ in tetragonal phase at room temperature for different operating frequencies $f=\omega / \omega_{0}$ shows the improvement of the MPB upon decreasing the operating frequency.

The other curves in Fig. 6(a) show that both the dynamic value of $\left|\chi_{z y y}^{S H G}\right|$ around the MPB and the corresponding value of $\beta^{*}$ are functions of the operating frequency. Particularly, at $f=0.3$ (curve $i i$ ), the maximum value of $\left|\chi_{z y y}^{\mathrm{SHG}}\right|$ is found to be around $4.8 \times 10^{-3} \mathrm{mv}^{-1}$ at 
$\beta^{*} \simeq 1.2$. At $f=0.5$ (curve iii), the maximum value of $\left|\chi_{z y y}^{S H G}\right|$ was found to be around $3.5 \times 10^{-3} \mathrm{mv}^{-1}$ at $\beta^{*} \simeq 1.5$. At $f=0.6$ (curve $i v$ ), the maximum value of $\left|\chi_{z y y}^{\text {SHG }}\right|$ was found to be around $1.5 \times 10^{-3} \mathrm{mv}^{-1}$ at $\beta^{*} \simeq 1.7$. At $f=0.7$ (curve $v$ ), the maximum value of $\left|\chi_{z y y}^{\mathrm{SHG}}\right|$ was found to be around $0.4 \times 10^{-3} \mathrm{mv}^{-1}$ at $\beta^{*} \simeq 2$. Therefore, a systematic increase of the operating frequency $\omega=f \omega_{0}$ results in a systematic decrease of the maximum value of $\left|\chi_{z y y}^{S H G}\right|$ and a systematic increase of the value $\beta^{*}$ too. Thus the dynamic $\left|\chi_{z y y}^{S H G}\right|$ assumes higher values at lower frequencies. The peaks exhibited by $\left|\chi_{z y y}^{\mathrm{SHG}}\right|$ can be viewed clearly upon plotting both the real and imaginary parts of the complex $\chi_{z y y}^{S H G}$ at single operating frequency $f=\omega / \omega_{0}=0.1$ as shown in Fig. $6(\mathrm{~b})$. The graph shows that the single pole exhibited by $\left|\chi_{z y y}^{S H G}\right|$ nearly at $\beta^{*} \approx 1$ results from the resonance-like response exhibited by both real and imaginary parts of the complex $\chi_{z y y}^{S H G}$. The values obtained in Fig. 6(a) are typical for SHG in $\mathrm{BaTiO}_{3}$ (Murgan et al., 2004). However, the maximum value of $\chi_{z y y}^{\text {SHG }}$ obtained in Fig. $6(\mathrm{~b})$ is dramatically increased $\left(2.5 \times 10^{-2} \mathrm{mV}^{-1}\right)$ at such low frequency. This value is higher than the typical values of $\mathrm{BaTiO}_{3}$ by about two to three order of magnitude. Needless to say that the SHG value in ferroelectrics is initially very large (typically $\chi^{(2)} \simeq 10^{-11} \mathrm{mV}^{-1}$ ) compared with the order of magnitude for typical dielectric or semiconductors in the nonresonant region. This is not surprising for resonant effects in highly polarizable materials such as ferroelectrics. The TO mode softening may be explained as a result of the well-known phenomenon of LO-TO splitting, that is, the shift in frequency between longitudinal optical and transverse optical phonons at the Brillouin zone centre (Waser et al., 2006). In this case, the softening of the TO modes is caused by a partial compensation of the long-range electric fields on one hand and the short-range lattice elastic forces on the other hand.

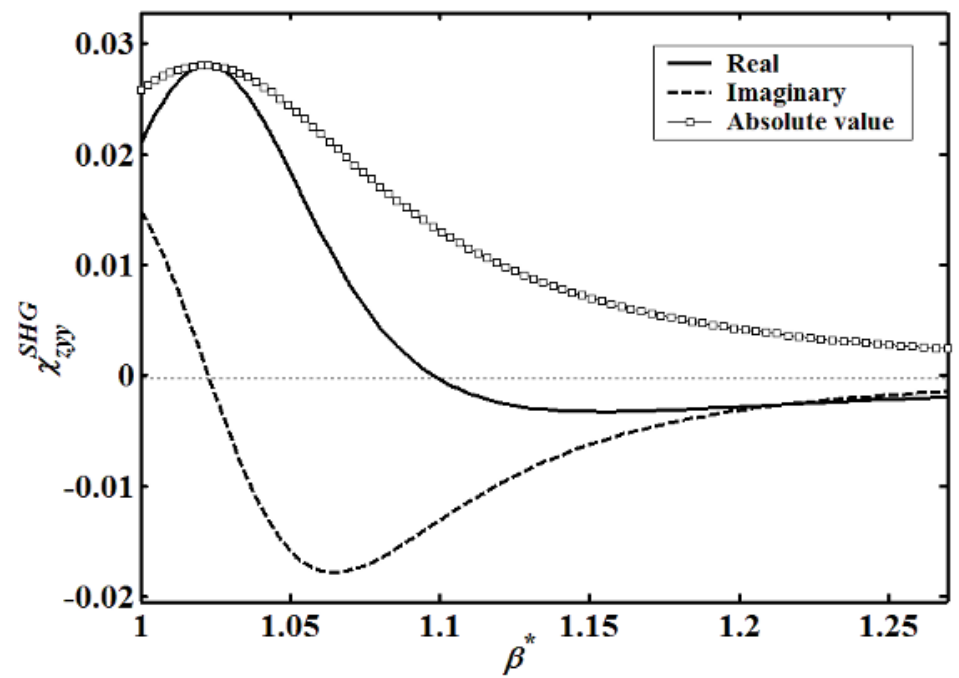

Fig. 6. (b) The real and imaginary part of the nonlinear dynamic dielectric susceptibility $\chi_{z y y}^{(2) S H G}(-2 \omega ; \omega, \omega)$ versus $\beta^{*}=\beta_{2} / \beta_{1}$ in tetragonal phase at room temperature and at operating frequency $f=\omega / \omega_{0}=0.1$. 


\section{Morphotropic phase boundary in third-order nonlinear susceptibility}

The expressions for the third-order nonlinear susceptibility elements also consist of products of linear response functions. Table 2 shows the dynamic nonlinear susceptibility elements for the third-harmonic generation (THG) is also expressed in terms of the linear response functions $\sigma(n \omega)$ and $s(n \omega)$. The integer number $n$ may assume one, two or three. The elements in Table 2 may be written in terms of the TO and LO phonon frequencies following the same procedure describes in the previous chapter. For example, the third-harmonic element $\chi_{z y y z}^{\text {THG }}$ in Eq. (17) may be written as;

$$
\begin{aligned}
\tilde{\chi}_{z y y z}^{(3) T H G} & =\frac{-\beta_{2}}{3 \varepsilon_{0}^{3} M^{4}\left[\frac{\Theta(3 \omega)}{M}-\omega_{L O}^{2}\right]\left[\frac{\Theta(\omega)}{M}-\omega_{L O}^{2}\right]} \\
& \times\left\{2 \frac{\beta_{2} \omega_{L O}^{2}}{\beta_{1}\left[\frac{\Theta(2 \omega)}{M}+\omega_{T O}^{2}\right]\left[\frac{\Theta(\omega)}{M}+\omega_{T O}^{2}\right]^{2}}+3 \frac{\omega_{L O}^{2}}{\left[\frac{\Theta(2 \omega)}{M}-\omega_{L O}^{2}\right]\left[\frac{\Theta(\omega)}{M}+\omega_{T O}^{2}\right]^{2}}+\frac{1}{\left[\frac{\Theta(\omega)}{M}+\omega_{T O}^{2}\right]^{2}}\right\}
\end{aligned}
$$

The linear response functions for the second-harmonics $\sigma(2 \omega) s(2 \omega)$ exists in Eq. (17) is responsible for the appearance of the function argument $\Theta(2 \omega)$ in Eq. (34) where $\Theta(2 \omega)=-4 M \omega^{2}-i 2 \Gamma \omega$. The function argument $\Theta(2 \omega)$ is intrinsic part of the $\chi^{(3)}$ response and they do not result from cascade processes in which two second-order process follow one another in time (Murgan et al., 2002). In nonlinear optics, a cascaded THG could arise from SHG followed by sum-frequency generation, or symbolically $\omega+\omega \rightarrow 2 \omega$, then $\omega+2 \omega \rightarrow 3 \omega$. The element $\chi_{z y y z}^{\text {THG }}$ in Eq. (34) (apart from the prefactors and the LO modes) include two terms related to the TO modes. The first term include a TO mode propagating at the input frequency $\omega$ while the second term include a TO mode propagating at $2 \omega$. Therefore, the element $\chi_{\text {zyyz }}^{\text {THG }}$ is expected to assume a double peak behavior which may lead to a complicated enhancement effects at two different values of $\beta^{*}$. In the static limit, Eq. (34) reduces to the following form;

$$
\tilde{\chi}_{z y y z z}^{(3) T H G}=\frac{-2 \beta_{2}}{3 \varepsilon_{0}^{3} M^{4} \omega_{T O}^{4} \omega_{L O}^{4}}\left[\frac{\beta_{2} \omega_{L O}^{2}}{\beta_{1} \omega_{T O}^{2}}-1\right]
$$

The former equation clearly indicates that the value of $\tilde{\chi}_{z y y z}^{(3) T H G} \rightarrow \infty$ when the value of $\omega_{\text {TO }} \rightarrow 0$ at the MPB.

A plot of $\chi_{z y y z}^{\text {THG }}$ versus $\beta^{*}$ is shown in Fig. 7(a). In the dynamic case, both the real (The - $\bullet-$ curve) and the imaginary parts (The -o- curve ) of $\chi_{z y y z}^{\text {THG }}$ assume a double peak behavior at two different values of $\beta^{*}$ as predicted by Eq. (34). In Fig. $7(\mathrm{~b})$, the value $\left|\chi_{z y y z}^{\text {THG }}\right|$ is plotted versus $\beta^{*}$ for different operating frequency. The main feature of these curves is that they all display a double peak response at certain value of $\beta^{*}$ as predicted by Eq. (34). The value of $\chi_{z y y z}^{\text {THG }}$ is generally increased upon decreasing the operating frequency $f$. As in the second-order case, this is due to the softening of the TO mode which results in a direct increment of $\chi_{z y y z}^{\text {THG }}$. 


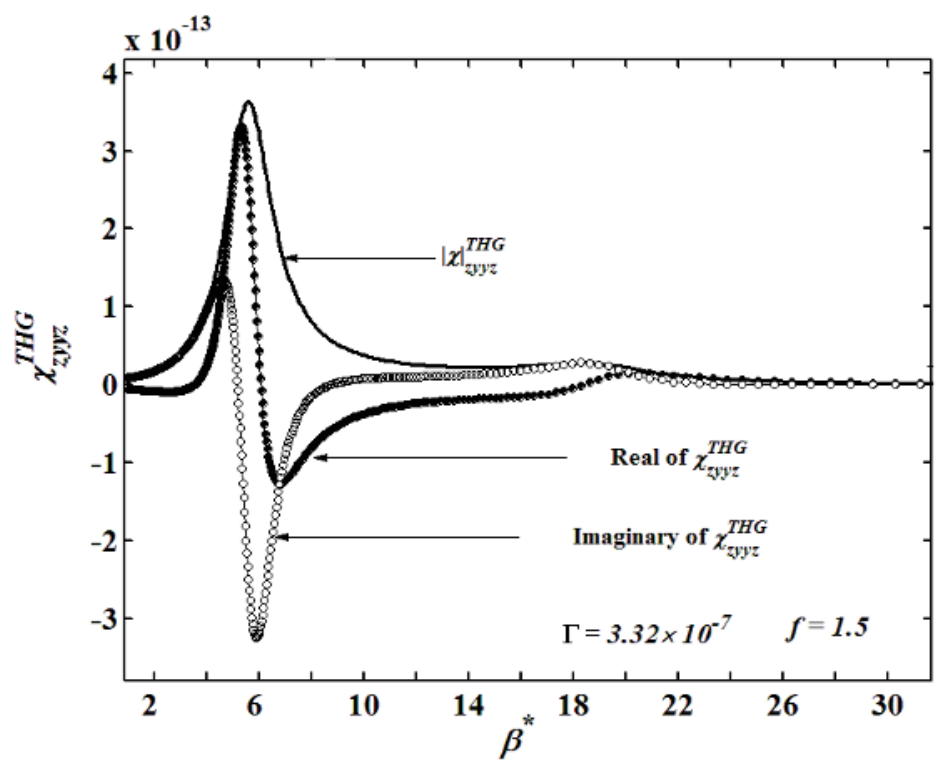

Fig. 7. (a) The third-order nonlinear susceptibility tensor element $\chi_{z y y z}^{\text {THG }}$ versus $\beta^{*}=\beta_{2} / \beta_{1}$ in tetragonal phase at room temperature for operating frequencies $f=1.5$ and for the static case at $f=0$.

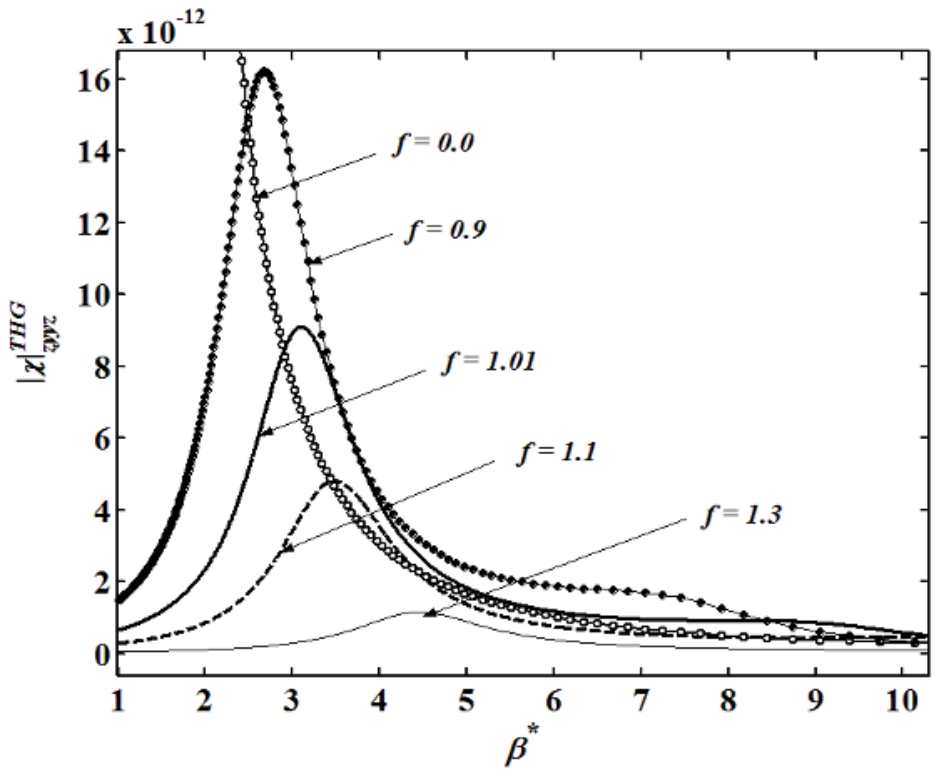

Fig. 7. (b) The absolute value nonlinear dielectric susceptibility $\left|\chi_{z y y z}^{\text {THG }}\right|$ versus $\beta^{*}=\beta_{2} / \beta_{1}$ in tetragonal phase at room temperature for different operating frequencies $f=\omega / \omega_{0}$. 
For example, at $f=1.3$ (thin solid curve), the maximum value of $\left|\chi_{z y y z}^{\text {THG }}\right|$ is found to be around $1.15 \times 10^{-12} \mathrm{~m}^{2} \mathrm{v}^{-2}$ at $\beta^{*} \simeq 4.5$. At $f=1.1$ (the dashed curve) the maximum value of $\left|\chi_{z y y z}^{\text {THG }}\right|$ is $4.8 \times 10^{-12} \mathrm{~m}^{2} \mathrm{v}^{-2}$ at $\beta^{*} \simeq 3.5$. At $f=1.01$ (solid curve), the maximum value of $\left|\chi_{z y y z}^{\text {THG }}\right|$ increases to $9.1 \times 10^{-12} \mathrm{~m}^{2} \mathrm{v}^{-2}$ at $\beta^{*} \simeq 3$. At $f=0.9$ (the $-\bullet$ - curve), the maximum value of $\left|\chi_{z y y z}^{\text {THG }}\right|$ further increases to $1.61 \times 10^{-11} \mathrm{~m}^{2} \mathrm{v}^{-2}$ at $\beta^{*} \simeq 2.7$. In the static case with $f=0$ (the -ocurve), the value of $\left|\chi_{z y y}^{\text {SHG }}\right|$ diverges at $\beta^{*}=1$ as predicted by Eq. (35). From the numerically generated data, the typical value of the dynamic $\chi^{(3)}$ in $\mathrm{BaTiO}_{3}$ is found to lie within the range of $10^{-13}$ to $10^{-16}$ depending on the operating frequency. Similar values of $\chi^{\text {THG }}$ for $\mathrm{BaTiO}_{3}$ are found by Murgan et al., (2002). These values for THG in ferroelectrics are very large compared with typical semiconductors or dielectrics. For example, the $\chi^{\text {THG }}$ for GaAs is about $6.7 \times 10^{-19} \mathrm{~m}^{2} \mathrm{v}^{-2}$ while it is in the range of $10^{-22}-10^{-20}$ for Glass (Eaton, 1991). From our numerically generated data, the value of $\chi^{(3)}$ in $\mathrm{BaTiO}_{3}$ at its peak is increased by two or three orders of magnitude in comparison with the values far from its peak.

\section{Conclusion}

In this chapter we have examined the behavior of both linear and nonlinear dielectric susceptibility as a function the free energy parameters for different operating frequencies. Both dynamic and static dielectric susceptibility is examined. Within the free-energy formulation, the material-dependent nonlinear coefficients $\beta_{1}$ and $\beta_{2}$ may be assumed as function of the molar composition. Using both the free energy and Landau-Khalatnikov equation, the nonlinear dielectric susceptibility is written as a product of the linear response functions. This form of dielectric susceptibility is transformed into an alternative form that shows the explicit dependence on the transverse-optical (TO) and longitudinaloptical (LO) modes. The dielectric susceptibility is then investigated within the concept of the ferroelectric soft-mode with normal frequency $\omega_{\text {TO }}$ where the material parameters $\beta_{1}$ and $\beta_{2}$ enters on the same footing as temperature. The divergence of the static dielectric susceptibility at the MPB occurs when the ferroelectric soft-mode becomes zero as a result of $\beta_{1} \rightarrow \beta_{2}$ or $T \rightarrow T_{c}$. Most dielectric susceptibility elements are systematically enhanced upon decreasing the operating frequencies. In the vicinity of the MPB, the low-frequency limit linear dielectric susceptibility is increased by one to two orders of magnitude in comparison with the non-MPB value of the static dielectric susceptibility. For secondorder NL elements, the dynamic NL susceptibility may assume single or double pole response. This actually depends on whether the dielectric susceptibility include a softmode corresponding to the input frequency $\omega$ or two soft-modes corresponds to both $\omega$ and $2 \omega$. We found that the second-order NL process is enhanced by two orders of magnitude in the vicinity of the MPB in comparison with the non-MPB values. We have also demonstrated the systematic enhancement of the dynamic nonlinear susceptibility upon increasing the thermodynamic temperature of the material towards the Curie temperature. Within the third-order process, we have investigated both the THG and the intensity-dependant (IP) susceptibility. We have found that certain elements within the THG process assumes a triple-response at three different values of $\beta^{*}$. This phenomena occurs if the NL susceptibility element includes three different soft-modes frequencies 
corresponds to $\omega, 2 \omega$ and $3 \omega$. Other elements within the third-order NL process may assume single or double pole in their dynamic response. As in the second-order process, the third order process is enhanced at the MPB by two to three orders of magnitude. All nonlinear elements are found to diverge in the static limit upon approaching $\beta^{*}=1$. It should be noted that we have investigated the MPB within the tetragonal phase where $\beta_{2}>\beta_{1}>0$, it will be very interesting to study the MPB for the rhombohedral phase within the free energy formalism. Finally we note that this calculation is important for designing a new material with higher nonlinearity for technological applications. This is achieved by investigating the MPB where the dielectric response is maximized.

\section{Acknowledgement}

The authors wish to express their sincere gratitude to Prof Y. Ishibashi and Prof D. R. Tilley for their fruitful discussion and support especially during their collaboration period with Universiti Sains Malaysia as visiting scientists.

\section{References}

Ahart M., Somayazulu M., Cohen R. E., Ganesh P., Dera P., Mao H-k, Hemley R. J., Ren Y., Liermann P. and Wu Z. Nature 451, 545 ( 2008).

Amin A., Newnhan R.E., and Cross L.E., Phys. Rev. B 341595 (1986).

Bellaiche L. and Vanderbilt D., Phys. Rev. Lett. 831347 (1999).

Cao W., and Cross L.E., Phys. Rev. B 474285 (1993).

Cohen, R. E., Nature 441, 941 (2006).

Cox D. E., Noheda B., Shirane G., Uesu Y., Fujishiro K., and Yamada Y., Appl. Phys. Lett. 79, 400 (2001)

Cross L., Ferroelectrics 76, 241 (1987).

Cross L., Ferroelectrics 151, 305 (1994).

Eaton D. F., Science, New Series, Vol. 253, No. 5017, 281 (1991).

Fu H., Cohen R., Nature 403, 281 (2000).

Glass A. M., Science, New Series, 235(4792) 1003 (1987).

Guo R., Cross L. E., Park S-E., Noheda B., Cox D. E., and Shirane G., Phys. Rev. Lett. 84, 5423 (2000).

Haas C., Phys. Rev. 140, A863 (1965).

Haun M. J., Furman E., Jang S.J., and Cross L.E., Ferroelectrics 9913 (1989).

Ibrahim A-B M. A., Tilley D. R., and Osman J., Ferroelectrics, 355 (1) 140 (2007).

Ibrahim A-B M. A., Tilley D. R., and Osman J., The Euro. Phys. J. B., 63(2) 1434 (2008). "Ferroelectric Optics: Optical bistability in Nonlinear Kerr ferroelectric materials"

Ibrahim A-B M. A., Rahman M. K. A., and Osman J., a chapter in book, "Ferroelectrics", Coondoo I., (Ed), ISBN: 978-953-307-439-9, InTech, (2010), Available from: http://www.intechopen.com/articles/show/title/ferroelectric-optics-opticalbistability-in nonlinear-kerr-ferroelectric-materials-

Ishibashi Y., and Orihara H., Ferroelectrics 156, 185 (1994).

Ishibashi Y., and Iwata M., Jpn. J. Appl. Phys. 37, L985 (1998).

Ishibashi Y., and Iwata M., J. Phys. Soc. Jpn. 68, 1353 (1999a).

Ishibashi Y., and Iwata M., Jpn. J. Appl. Phys. 38, 800 (1999b) 
Ishibashi Y., Iwata M., J. Phys. Soc. Jpn. 68, 1353 (1999c)

Ishibashi Y., and Iwata M., Jpn. J. Appl. Phys. 38, 1454 (1999d)

Ishibashi Y., Ferroelectrics 264, 197 (2001).

Ishibashi Y., Iwata M., J. Phys. Soc. Jpn. 72, 1675 (2003)

Iwata M., Orihara H., and Ishibashi Y., Ferroelectrics 266, 57 (2002a)

Iwata M., Araki T., Maeda M., Suzuki I., Ohwa H., Yasuda N., Orihara H., and

Ishibashi Y., Jpn. J. Appl. Phys. 41, $7003\left(2002^{\mathrm{b}}\right)$

Iwata M., Ishibashi Y., J. Phys. Soc. Jpn. 72, 2834 (2003)

Iwata M., and Ishibashi Y., In "Ferroelectric thin films" by Okuyama M., and Ishibashi Y., Springer -Verlag Berlin Heidelberg, (2005).

Jaffe B., Roth R. S. and Marzullo S., J. Appl. Phys. 25, 809 (1954)

Jaffe B., Crook J. W. J., and Jaffe H.L., "Piezoelectric Ceramics", Academic Press,

London (1971).

Kakegawa K., 1Department of Applied Chemistry, Faculty of Engineering, Chiba University,

Katayama, I., Shimosato, H., Ashida, M., Kawayama, I., Tonouchi, M., Itoh, T., “Conference on Lasers and Electro-Optics CLEO", Pages 1-2 (2007).

Kittel C., "Introduction to Solid State Physics", 7th ed. New York: Wiley, (1995).

Kuwata J., Uchino K., and Nomura S., Ferroelectrics 37, 579 (1981)

Kuwata J., Uchino K., and Nomura S., Jpn. J. Appl. Phys. 211298 (1982).

Mishra S. K., and Pandey D., Appl. Phys. Lett. 691707 (1996)

Mitsui T., Tatsuzaki I., and Nakamura E., "An Introduction to the Physics of Ferroelectrics", Gordon and Breach, London, (1976)

Murgan R., Tilley D. R., Ishibashi Y., Webb J. F., and Osman J., J. Opt. Soc. Am. B 192007 (2002).

Murgan R., Tilley D. R., Ishibashi Y., and Osman J., J. Fizik Malaysia. 23, 1 (2002a).

Murgan R., Razak F., Tilley D. R., Tan T. Y., Osman J., and Halif M. N. A., Computational Material Science. 30468 (2004).

Newnham R., Mater. Res. Soc. Bull. 2220 (1997).

Noheda B., Cox D. E., Shirane G., Gonzalo J.A., Cross L.E., and Park S. E., Appl. Phys. Lett. 74, 2059 (1999).

Noheda B., Gonzalo J., Cross L., Guo R., Park S.E., Cox D., Shirane G.: Phys. Rev. B 61, 8687 (2000a)

Noheda B., Cox D., Shirane G., Guo R., Jones B., Cross L.: Phys. Rev. B 63, 014103, (2000 b)

Osman J., Ishibashi Y., and Tilley D. R., Jpn. J. of Appl. Phys, 37, 4887 (1998a).

Osman J., Ishibashi Y., Lim S-C., and Tilley D. R., J. of Kor. Phys. Soc. 32, S446 (1998b). Razak F.1, Webb J. F., Murgan R., Ishibashi Y., Osman J., Ferroelectrics, 273 179, (2002).

Saghi-Szabo G., Cohen R., and Krakauer H., Phys. Rev. B 59, 12771 (1999).

Sawaguchi E., J. Phys. Soc. Jpn. 8, 615 (1953).

Shirane G., Suzuki K., Jpn. J. Appl. Phys. 7333 (1952).

Sirenko A. A., Bernhard C., Golnik A., Clark A. M., Hao J., Si W, Nature, 404, 373 ( 2000 ).

Vanderbilt D., and Cohen M.: Phys. Rev. B 63, 94108 (2001)

Waser C. Böttger U., and Tiedke S., "Polar Oxides: Properties, Characterization, andImaging", $4^{\text {th }}$ edition, Weinheim, Wiley-VCH, (2005).

Wilkinson A.P., Xu J., Pattanaik S., and Billinge J.L., Chem. Mat. 103611 (1998).

Yamamoto T. and Ohashi S., Jpn. J. Appl. Phys., 345349 (1995). 
Yamashita Y., Jpn. J. Appl. Phys., 335328 (1994).

Zhang S., Dong X., and Kojima S.. Jpn. J. Appl. Phys. 362994 (1997). 


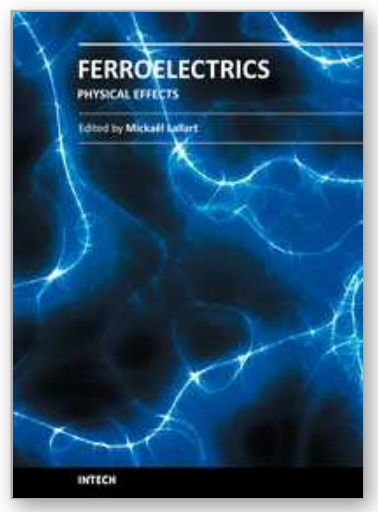

\author{
Ferroelectrics - Physical Effects \\ Edited by Dr. MickaÃ «l Lallart
}

ISBN 978-953-307-453-5

Hard cover, 654 pages

Publisher InTech

Published online 23, August, 2011

Published in print edition August, 2011

Ferroelectric materials have been and still are widely used in many applications, that have moved from sonar towards breakthrough technologies such as memories or optical devices. This book is a part of a four volume collection (covering material aspects, physical effects, characterization and modeling, and applications) and focuses on the underlying mechanisms of ferroelectric materials, including general ferroelectric effect, piezoelectricity, optical properties, and multiferroic and magnetoelectric devices. The aim of this book is to provide an up-to-date review of recent scientific findings and recent advances in the field of ferroelectric systems, allowing a deep understanding of the physical aspect of ferroelectricity.

\title{
How to reference
}

In order to correctly reference this scholarly work, feel free to copy and paste the following:

Abdel-Baset M. A. Ibrahim, Rajan Murgan, Mohd Kamil Abd Rahman and Junaidah Osman (2011). Morphotropic Phase Boundary in Ferroelectric Materials, Ferroelectrics - Physical Effects, Dr. Micka $\tilde{l}$ Lallart (Ed.), ISBN: 978-953-307-453-5, InTech, Available from: http://www.intechopen.com/books/ferroelectricsphysical-effects/morphotropic-phase-boundary-in-ferroelectric-materials

\section{INTECH}

open science | open minds

\section{InTech Europe}

University Campus STeP Ri

Slavka Krautzeka 83/A

51000 Rijeka, Croatia

Phone: +385 (51) 770447

Fax: +385 (51) 686166

www.intechopen.com

\section{InTech China}

Unit 405, Office Block, Hotel Equatorial Shanghai

No.65, Yan An Road (West), Shanghai, 200040, China 中国上海市延安西路65号上海国际贵都大饭店办公楼 405 单元

Phone: +86-21-62489820

Fax: +86-21-62489821 
(C) 2011 The Author(s). Licensee IntechOpen. This chapter is distributed under the terms of the Creative Commons Attribution-NonCommercialShareAlike-3.0 License, which permits use, distribution and reproduction for non-commercial purposes, provided the original is properly cited and derivative works building on this content are distributed under the same license. 